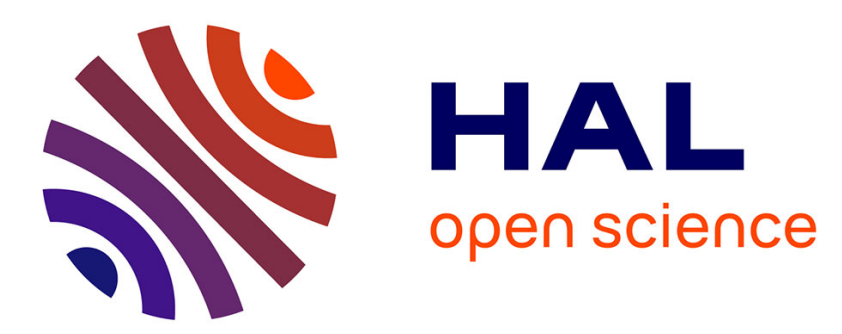

\title{
Degree of polarization of luminescence from GaAs and InP as a function of strain: a theoretical investigation
}

Daniel T Cassidy, Jean-Pierre Landesman

\section{To cite this version:}

Daniel T Cassidy, Jean-Pierre Landesman. Degree of polarization of luminescence from GaAs and $\mathrm{InP}$ as a function of strain: a theoretical investigation. Applied optics, 2020, 59 (18), pp.5506. 10.1364/AO.394624 . hal-03029987

\section{HAL Id: hal-03029987 https://hal.science/hal-03029987}

Submitted on 29 Nov 2020

HAL is a multi-disciplinary open access archive for the deposit and dissemination of scientific research documents, whether they are published or not. The documents may come from teaching and research institutions in France or abroad, or from public or private research centers.
L'archive ouverte pluridisciplinaire HAL, est destinée au dépôt et à la diffusion de documents scientifiques de niveau recherche, publiés ou non, émanant des établissements d'enseignement et de recherche français ou étrangers, des laboratoires publics ou privés. 


\title{
Degree of polarization of luminescence from GaAs and InP as a function of strain: a theoretical investigation
}

\author{
Daniel T. Cassidy ${ }^{1,3}$ And JeAn-PIERRE LANDEsman ${ }^{2,4}$ \\ ${ }^{1}$ McMaster University, Department of Engineering Physics, Hamilton, ON, L8S 4L7, Canada \\ ${ }^{2}$ Univ Rennes, CNRS, IPR - UMR 6251, F-35000 Rennes, France \\ 3 cassidy amcmaster.ca \\ ${ }^{4}$ jean-pierre. Iandesmaneuniv-rennes1. fr
}

\begin{abstract}
:
Experimentally it is known that the degree of polarization (DOP) of luminescence is a sensitive function of strain in III-V materials. It has been assumed that DOP $=-K_{e}\left(e_{1}-e_{2}\right)$ and that the rotated degree of polarization $(\mathrm{ROP})=2 K_{e} e_{6}$, where $K_{e}$ is a positive calibration constant, $e_{1}$ and $e_{2}$ are the normal components of strain along perpendicular ' 1 ' and ' 2 ' directions, and $e_{6}=e_{12}$ is the tensor shear strain. $K_{e}$ has been measured experimentally for GaAs and InP.

In this paper, the results of a simple analytic determination of expressions for DOP as a function of strain are presented. Given the wide ranges reported for the strain deformation potentials $b$ and $d$, it is not possible to give definitive and meaningful numerical values for expressions for DOP and $K_{e}$. However, the sensitivity of DOP to strain suggests that it might be possible to design simple experiments to provide accurate values for the deformation potentials.

The $b$ and $d$ deformation potentials might not be independent. For the results presented here
\end{abstract}

and in the limit of isotropic material, an isotropic result for the DOP is found if $d=\sqrt{ } 3 b$.

\section{Introduction}

The degree of polarization (DOP) of luminescence from III-V materials, such as GaAs and InP and their alloys, is a sensitive function of strain [1-5]. DOP measurements [6-10] have been applied to investigations of bonding strain $[2,4,11-14]$, to understanding spectral properties of lasers [15], to estimation of strain and photo-elastic effects owing to over-layers [16-18], to identify dislocations [3,5], to reliability investigations [19], to effects of strain on the operation and yield of DFB lasers [20,21], to growth related issues [22-25], and to characterize high power lasers $[10,13,19,26]$.

Recently, Mokhtari et al. [27] used the DOP of photoluminescence technique to investigate the occurrence of mechanical stress in vertical cavity surface emitting lasers (VCSELs), designed for emission at $850 \mathrm{~nm}$, in relation to the lateral oxidation process used to control current injection and lateral optical confinement. Landesman et al. $[28,29]$ used the same technique to quantify the anisotropic stress introduced in InP or GaAs substrates by the presence of thin and narrow dielectric stripes on (100) surfaces. These stripes can play the role of hard masks for deep reactive ion etching of InP or GaAs. Landesman et al. [29] demonstrated, using the DOP of photoluminescence technique, that the reactive ion etching process itself can generate highly anisotropic stress in the etched material, independent of the role of the hard mask layer.

Yan and Zhou [30] used DOP measurements to investigate bonding strain for p-up and pdown superluminescent diode lasers. Fouchier et al. [31] extended the DOP technique to cathodoluminescence (CL) measurements and demonstrated a strain sensitivity of $10^{-5}$ and spatial resolution of the order of $100 \mathrm{~nm}$ on InP samples. Chen et al. [32] used DOP measurements in 
their investigations of $\mathrm{ZnO}$. Lin et al. [33] and Peloso et al. [34] applied polarization resolved photoluminescence to the characterization of photovoltaic silicon wafers. Winterfeldt et al. [35] used degree of polarization measurements to aid in the investigation of the factors limiting the lateral beam parameter of 9xx nm high-power broad-area lasers. Hempel et al. [36] made measurements on high-power lasers and provided a comparison of strain measurements by photoluminescence, Raman, and DOP of electroluminescence. Matsui et al. [37] used results from DOP measurements and finite elements simulations of strain in their investigations of tuneable VCSELs. Pittroff et al. [38] used DOP measurements under the $200 \mu \mathrm{m}$ wide stripe of high-power III-V lasers to estimate the strain caused by different bonding approaches and found no influence of mounting stresses on degradation for an optimized soldering process.

The DOP of photoluminescence (PL) is a local probe technique [39], much like photocurrent spectroscopy [40] and cathodoluminescence [29,31] are local probe techniques. However, DOP measurements use the integrated luminescence [7]. This makes DOP measurements potentially faster, simpler, and more sensitive than other local probe techniques that require spectral resolution of the luminescence.

Strain estimation by analysis of the DOP of luminescence can be applied to both bulk and nano-structures [41,42]. Emission from nano-structures such as quantum wells (QWs) is inherently polarized. Strain adds to the DOP of QWs [41]. The DOP for PL from the normal to the plane of InGaAs QWs was found to be set by strained bonds across the interfaces of the QWs [42]. Virtual force field (VFF) simulations revealed that the DOP of the PL was sensitive to the assumed bond strengths for the binaries existing in the monolayers at the interfaces [43].

The spatial resolution for DOP measurements derived from PL is essentially diffraction limited. Spatial resolution can be improved by using an electron beam to create luminescence, i.e., cathodoluminescence $[29,31]$. The noise level for DOP measurements is typically $0.1 \%$, which translates roughly to a strain-equivalent noise level of $\approx 10^{-5}[4,7]$. The translation from DOP to strain depends on an assumed functional dependence of DOP on strain and a calibration constant. It was assumed that the DOP is proportional to the difference of two normal components of stress or of strain, and the calibration constant $K_{e}$ was experimentally determined [2,3,7]. It was demonstrated that the difference of normal components of strain fit finite element method (FEM) simulations of an experiment better than the difference of two normal components of stress, and the calibration constant for $\mathrm{InP}$ was obtained from fits of these simulations to data [7]. The limiting noise source for DOP measurements using a rotating polarizer and a mechanical chopper was investigated [44] and was found to a multiplicative noise from a beat frequency between the polarizer and chopper frequencies.

In this paper the functional dependence of DOP on strain for III-V materials is investigated by estimating the band-to-band transition rates for two orthogonal polarizations by using expressions for the strain-dependant energy dispersion of the bands. The bands are assumed to be parabolic. Boltzmann statistics and selection rules are used to find the DOP of luminescence. This leads to an expression for the DOP as a function of strain and the calibration constant $K_{e}$ is obtained from this expression.

T. B. Bahder gave the matrix elements for an $8 \times 8 \boldsymbol{k} \cdot \boldsymbol{p}$ Hamiltonian for a uniformly strained zinc-blende crystal [45, June 1990], with corrections to the results for the spin-orbit terms published in October 1992 [46]. In January 1992 Bahder published approximate, analytic dispersions relations for strained zinc-blende crystals [47]. These expressions are based on perturbative expansions of the $8 \times 8 \boldsymbol{k} \cdot \boldsymbol{p}$ Hamiltonian, which reduced the dimensionality and thus made it easier to find the eigenvalues. Generally the perturbative expansions are correct to linear in strain and fourth-order in wave vector [47, RHS, pg 1630]. In addition, the eigenvalue problem was linearized by using the average value of the diagonal elements for the energy in the denominator of the Löwdin perturbation [47, LHS, pg 1640]. The $8 \times 8 \boldsymbol{k} \cdot \boldsymbol{p}$ Hamiltonian includes strain-induced valence- and conduction-band mixing and is based on the usual second-order 
Löwdin perturbation theory. The Luttinger four-band model with strain is recovered from Bahder's eight band model [45] if the asymmetry parameter $B$ is set to zero and the band gap and spin-orbit splitting are taken to be large [45, Sec. III].

Bahder compared his approximate, analytic results to numerical computations of eigenvalues for the $8 \times 8 \boldsymbol{k} \cdot \boldsymbol{p}$ Hamiltonian and found them to be accurate for small $|\boldsymbol{k}|$, with small $|\boldsymbol{k}|$ being $<0.33 \mathrm{~nm}^{-1}$ [47]. Bahder notes in Ref. 17 of [47] that the original Hamiltonian neglects terms that are linear in $\boldsymbol{k}$, which might be important, and accuracy might be compromised for small $\boldsymbol{k}$.

Bahder used scaled parameters; see [47, Ref. 20]. The same scaling is employed here and thus $k$ should be replaced with $k \times \sqrt{\hbar^{2} /\left(m_{o} q\right)}, A_{p}{ }^{2}$ should be replaced with $A_{p}{ }^{2} \times m_{0} q / \hbar^{2}$, and $P_{o}{ }^{2}$ should be replaced with $P_{o}{ }^{2} \times m_{o} q / \hbar^{2}$, where $q$ is used to convert to $\mathrm{eV}$. Physical and material constants and their symbols are defined in Appendix A.

Of interest to this work is that Bahder [45-47] did not specialize to biaxial strain, as would be found in strained layer epitaxy. The Hamiltonians that Bahder worked with have shear strain terms and thus analytic expressions contain shear strain terms.

\section{Approach}

We use Bahder's analytic expressions for the energy dispersion [47] and the $\boldsymbol{k}=0$ eigenfunctions to calculate the strain-dependent spontaneous emission for two orthogonal polarizations. Using the $\boldsymbol{k}=0$ eigenfunctions is an approximation to minimize the quantity of algebra that is required to find the calibration constant. It remains for corroboration to see if the approach is accurate.

The eigenfunctions are taken to be in a reference frame for $\boldsymbol{k}$ along a $z^{\prime}$ direction. The original eigenfunctions must be rotated from the crystal coordinate system, which has $x$ along [100], $y$ along [010], $z$ along [001], and $\boldsymbol{k}$ along the $z$ direction, to be in the $\boldsymbol{k}$-along- $z^{\prime}$ coordinate system.

Enders [48] notes that the Hamiltonian is at its simplest form when $\boldsymbol{k}$ is along the $z$ direction as some non-diagonal terms in the Hamiltonian are functions of $k_{x}$ and $k_{y}$, and $k_{x}$ and $k_{y}$ are zero for $\boldsymbol{k}$ along $z$. Any $\boldsymbol{k}$ along an arbitrary direction $z^{\prime}$ is related to the components in the original $z$ coordinate system by Euler rotations (see Appendix E). Enders also notes that a block diagonalisation of a Hamiltonian with $\boldsymbol{k}$ can be perturbed by the rotation of the coordinate system. Enders proposed selective neglect of small terms that perturb the block diagonalisation. One can avoid the problem of block diagonalisation by using Bahder's analytic approximations [47].

To minimize subscripts we write tensors in a Voigt notation. The strain tensor $[e]$ is then, in this notation,

$$
[e]=\left[\begin{array}{ccc}
e_{1} & e_{6} & e_{5} \\
e_{6} & e_{2} & e_{4} \\
e_{5} & e_{4} & e_{3}
\end{array}\right]=\left[\begin{array}{ccc}
e_{x x} & e_{x y} & e_{x z} \\
e_{x y} & e_{y y} & e_{y z} \\
e_{x z} & e_{y z} & e_{z z}
\end{array}\right],
$$

with trace given by $\operatorname{tr}([e])=e_{1}+e_{2}+e_{3}$.

Ostromek [49] used Bahder's [45,46] $8 \times 8 \boldsymbol{k} \cdot \boldsymbol{p}$ Kane Hamiltonian, which takes into account both the inversion asymmetry $B$ and the $k$-dependent spin-orbit interaction, to find through optimization a consistent set of band parameters for GaAs. Ostromek found the set of band parameters to be weakly dependent on the value of $A_{p}$, which couples the conduction band to higher lying bands. It was suggested $A_{p}=-14.7 \mathrm{eV} \AA^{2}$ with $E_{p}=29.112 \mathrm{eV}$ or $A_{p}=0$ with $E_{p}=22.827 \mathrm{eV} . B \neq 0$ as conduction band spin-splitting is observed, but Ostromek found inconsistent values for Kane non-parabolicity parameters with $B \neq 0$ [49, pg 14475]. We set $B=0$ and ignore any spin-dependent splitting of the conduction band.

Photoluminescence occurs near the band edge where $|\boldsymbol{k}|$ is small. We thus restrict interest to small $|\boldsymbol{k}|$. 


\subsection{Density of States}

Rather than calculating all possible transitions, we use an effective density of states that is a function of the effective masses. If a value for $|\boldsymbol{k}|$ is required, we use a value of $k=|\boldsymbol{k}|$ for which the spontaneous emission (i.e., luminescence) is a maximum.

We use Boltzmann statistics $\exp \left(-E / k_{B} T\right)$ and a parabolic band with a density of states $\rho(E) \propto \sqrt{E}$. The maximum value of $\sqrt{E} \exp \left(-E / k_{B} T\right)$ occurs at $E=k_{B} T / 2$. The maximum value of $k \exp \left(-k^{2} / k_{B} T\right)$ occurs at $k= \pm \sqrt{k_{B} T / 2}$. At $298 \mathrm{~K}, k_{B} T=25.7 \mathrm{meV}$.

For a parabolic band, an effective density of states $N_{e}$ can be defined such that the carrier density $n$ is given by

$$
n=N_{e} \exp \left(\left(E_{F}-E_{o}\right) / k_{B} T\right)=2\left(\frac{m_{e} k_{B} T}{2 \pi \hbar^{2}}\right)^{3 / 2} \times \exp \left(\left(E_{F}-E_{o}\right) / k_{B} T\right)
$$

with $E_{F}$ a quasi-Fermi energy and $E_{o}$ the band edge energy. This effective density of states is proportional to $m_{e}^{3 / 2}$ with $m_{e}$ an effective mass. The concept of an effective density of states can be extended to non-parabolic bands [50].

\section{Degree of Polarization (DOP)}

In the context of strain investigations, the degree of polarization (DOP) was defined to be $[1,7]$

$$
D O P_{\hat{\boldsymbol{h}} \times \hat{\boldsymbol{v}}}=\frac{L_{\hat{\boldsymbol{h}}}-L_{\hat{\boldsymbol{v}}}}{L_{\hat{\boldsymbol{h}}}+L_{\hat{\boldsymbol{v}}}}
$$

where $\hat{\boldsymbol{h}}$ and $\hat{\boldsymbol{v}}$ are two orthogonal unit vectors in the plane of the surface that is being measured, $L_{\hat{\boldsymbol{q}}}$ is the measured irradiance for light polarized along a direction given by the unit vector $\hat{\boldsymbol{q}}$, $\hat{\boldsymbol{q}}=\hat{\boldsymbol{h}}$ or $\hat{\boldsymbol{v}}$, and the normal to the surface under study is given by the vector cross product $\hat{\boldsymbol{h}} \times \hat{\boldsymbol{v}}$. Usually the directions $\hat{\boldsymbol{h}}$ or $\hat{\boldsymbol{v}}$ are chosen to correspond to a feature of interest of the sample, such as $\hat{\boldsymbol{h}}$ parallel to the layers for a measurement from a cleaved facet of a laser, or $\hat{\boldsymbol{v}}$ parallel to a stripe for measurements from a (001) surface.

We also defined an ROP or rotated degree of polarization to be $[3,7]$

$$
R O P_{\hat{\boldsymbol{h}}^{\prime} \times \hat{\boldsymbol{v}}^{\prime}}=\frac{L_{\hat{\boldsymbol{h}^{\prime}}}-L_{\hat{\boldsymbol{v}^{\prime}}}}{L_{\hat{\boldsymbol{h}^{\prime}}}+L_{\hat{\boldsymbol{v}^{\prime}}}}
$$

where the directions $\hat{\boldsymbol{h}}^{\prime}$ and $\hat{\boldsymbol{v}}^{\prime}$ are obtained by a -45 deg rotation about the normal to the surface, $\hat{\boldsymbol{h}} \times \hat{\boldsymbol{v}}$. Experimentally we determined that the DOP is proportional to the difference of the normal components of strain, $e_{h h}-e_{v v}$, and ROP is proportional to twice the tensor shear strain $2 e_{h v}$.

Given the definition of DOP as the ratio of the difference to sum, common factors cancel out and one need only retain relative terms. Thus to find an expression for $D O P_{\hat{\boldsymbol{h}} \times \hat{v}}$ one should need only the strain-dependent shifts in energy of the light-hole (LH) and heavy-hole (HH) bands, the effective masses, and the wave functions for the bands. The wave functions are needed to calculate the polarization resolved photoluminescence.

\subsection{Calibration Constant $\boldsymbol{K}_{\boldsymbol{e}}$}

The calibration constant $K_{e}$ is estimated by calculating values for the photoluminescences $L_{\hat{\boldsymbol{h}}}$ and $L_{\hat{v}}$. To simplify calculation of $K_{e}$, the photoluminescence is assumed to originate from the band edges. Thus $k^{4} \ll k^{2}$ and the bands can be assumed to be parabolic. The strain dependent shifts of the light-hole and heavy-hole bands are calculated using Bahder's analytic approximations [47]. An effective density of states and Boltzmann statistics are used to obtain the relative contributions to the photoluminescences. The carriers, which have crystal momentum $\boldsymbol{k}$, are assumed to be 
evenly divided amongst the two orthogonal directions $\hat{\boldsymbol{h}}$ and $\hat{\boldsymbol{v}}$. It is not necessary to attempt to calculate luminescences for carriers with $\boldsymbol{k}$ along $\hat{\boldsymbol{h}} \times \hat{\boldsymbol{v}}$ as this is the direction of observation and electromagnetic radiation is a transverse wave - see Appendix C. Momentum matrix elements with $\boldsymbol{p}$ along the direction of polarization are used to calculate the relative rates of emission of the polarized luminescences. It is assumed that the values calculated along two directions $(\boldsymbol{k}$ along $\hat{\boldsymbol{h}}$ and $\hat{\boldsymbol{v}}$ ) are sufficient to describe accurately $L_{\hat{\boldsymbol{h}}}$ and $L_{\hat{\boldsymbol{v}}}$ (i.e., no integration over contributions from different directions, as in many cases the integrals are elliptic integrals). For calculations the $z$-direction is aligned with the crystal momentum of the carriers involved in the calculation and rotation of the coordinate system is used as necessary to obtain results in the observer's frame of reference.

Using a $2 \times 2 \mathrm{~L}-\mathrm{K}$ Hamiltonian with biaxial strain, the band edge (i.e., $\boldsymbol{k}=0$ ) splitting of the light-hole and heavy-hole bands is given by $\Delta=2 Q_{e}= \pm b\left(\operatorname{tr}([e])-3 e_{3}\right)$ :

$$
H=\left[\begin{array}{cc}
-P+a \operatorname{tr}([e])-Q-Q_{e} & R+i S \\
R^{*}-i S^{*} & -P+a \operatorname{tr}([e])+Q+Q_{e}
\end{array}\right],
$$

since at the band edge and for biaxial strain (i.e., $e_{1}=e_{2}$ and the shears strain $e_{4}, e_{5}, e_{6}=0$ ), $P=Q=R=S=0$.

The standard L-K Hamiltonian with strain tends to be specialized to biaxial strain, as would be found in strained layer epitaxy [51, Eq. 4]. If the strain is not biaxial, then $R$ and $S$ are not zero at the band edge, and have real and imaginary components.

For arbitrary strain, the results obtained with a $2 \times 2 \mathrm{~L}-\mathrm{K}$ Hamiltonian and by Bahder [47] do not seem to be consistent.

Bahder states that his results reproduce, in the limit of no coupling to the conduction band or spin-orbit band, the results of Pikus and Bir [47, pg 1633]. Pikus and Bir used a Luttinger model that did not include mixing of the conduction and spin-orbit bands with the valence bands. We will work with the analytic approximations derived by Bahder [47].

\subsection{Conduction Band Dispersion}

Bahder's [47] expression for the conduction band dispersion $E_{c}$, neglecting strain and keeping only terms of order $k^{2}$, is given below. The effects of the strain on the conduction band are not critical in calculation of the DOP, as the effects of these shifts are common to all transitions and thus cancel out in the determination of the DOP, Eq. (3). In the expression for $E_{c}, A_{p}$ accounts for mixing with higher order bands [47]. Ostromek [49] found for GaAs that $A_{p}=0$ and $E_{p}=22.8$ or $A_{p}=-14.7$ and $E_{p}=29.1$. Vurgaftman et al. [52] recommend $E_{p}=28.8 \mathrm{eV}$, which would suggest $A_{p}=-14.7 \AA^{2}$.

$$
\begin{aligned}
E_{c} & =E_{g}+\left(k_{x}^{2}+k_{y}{ }^{2}+k_{z}^{2}\right)\left(\frac{1}{2}+A_{p} \frac{\left(10^{-10}\right)^{2} m_{o} q}{\hbar^{2}}+\frac{P_{o}^{2}\left(E_{g}+2 / 3 \Delta\right)}{E_{g}\left(E_{g}+\Delta\right)}\right) \\
& =1.5177+\left(k_{x}{ }^{2}+k_{y}{ }^{2}+k_{z}{ }^{2}\right)(0.5-1.916+8.9078) .
\end{aligned}
$$

The recommended value for the GaAs conduction band effective mass at $T=0$, in units of $m_{o}$, is $m_{c}=0.067$ [52]. With $A_{p}=0$ and $E_{p}=22.8$, the expression above for $E_{c}$ yields $m_{c}=0.0662$, and with $A_{p}=-14.7$ and $E_{p}=28.8$, the expression gives $m_{c}=0.0669$. Given the results obtained, for GaAs we use $A_{p}=-14.6$ and $E_{p}=28.8 \mathrm{eV}$. 


\subsection{Valence Band Dispersions}

Bahder's equation for the valence band energy as a function of $\boldsymbol{k}=\left(k_{x}, k_{y}, k_{z}\right)$ and strain [e] [47, Eq 9] is

$$
E_{v}=E_{v}^{(0)}+E_{v}^{(1)} \pm \sqrt{E_{k}+E_{k s}+E_{s}}
$$

with

$$
E_{v}^{(0)}=-\left(k_{x}^{2}+k_{y}^{2}+k_{z}^{2}\right)\left(\frac{P_{o}^{2}}{3 E_{g}}+\frac{\gamma_{1 m}}{2}\right)+O\left(k^{4}\right)
$$

and

$$
\begin{aligned}
E_{v}^{(1)} & =-a_{v} \operatorname{tr}([e])+\left(e_{1} k_{x}^{2}+e_{2} k_{y}^{2}+e_{3} k_{z}^{2}\right)\left(\frac{P_{o}{ }^{2}}{3 E_{g}}\left(2+\frac{a_{p}}{E_{g}}\right)-2 \frac{b \gamma_{2 m}}{\Delta_{s o}}\right) \\
& +\left(k_{x}^{2}\left(e_{2}+e_{3}\right)+k_{y}^{2}\left(e_{1}+e_{3}\right)+k_{z}^{2}\left(e_{1}+e_{2}\right)\right)\left(\frac{a_{p} P_{o}^{2}}{3 E_{g}{ }^{2}}+\frac{b \gamma_{2 m}}{\Delta_{s o}}\right) \\
& +\left(e_{4} k_{y} k_{z}+e_{5} k_{x} k_{z}+e_{6} k_{x} k_{y}\right)\left(\frac{4 P_{o}^{2}}{3 E_{g}}-2 \frac{\sqrt{3} d \gamma_{3 m}}{\Delta_{s o}}\right)
\end{aligned}
$$

and

$$
\begin{aligned}
E_{k} & =\left(k_{x}{ }^{2}+k_{y}{ }^{2}+k_{z}{ }^{2}\right)^{2}\left(\gamma_{2 m}+\frac{P_{o}{ }^{2}}{3 E_{g}}\right)^{2} \\
& +\left(k_{x}{ }^{2} k_{y}{ }^{2}+k_{x}{ }^{2} k_{z}{ }^{2}+k_{y}{ }^{2} k_{z}{ }^{2}\right)\left(\gamma_{3 m}-\gamma_{2 m}\right)\left(3 \gamma_{3 m}+3 \gamma_{2 m}+2 \frac{P_{o}{ }^{2}}{E_{g}}\right)
\end{aligned}
$$

Both $E_{v}^{(0)}$ and $E_{v}^{(1)}$ shift both the light-hole and the heavy-hole band, and do not contribute to valence band splitting and to DOP. $E_{k}$ is strain independent and contributes to the $\boldsymbol{k}$-dependant splitting between the light and heavy holes, i.e, to the different effective masses of the valence bands.

Bahder's $E_{k s}$ has a form [47, Eq. 13] that selects strain along a given $\boldsymbol{k}$, which leads to DOP:

$$
\begin{array}{r}
E_{k s}=b\left(\gamma_{2 m}+\frac{P_{o}^{2}}{3 E_{g}}\right)\left(\left(k_{x}{ }^{2}+k_{y}{ }^{2}+k_{z}{ }^{2}\right) \times \operatorname{tr}([e])-3 k_{x}^{2} e_{1}-3 k_{y}{ }^{2} e_{2}-3 k_{z}{ }^{2} e_{3}\right) \\
-2 \sqrt{3} d\left(\gamma_{3 m}+\frac{P_{o}{ }^{2}}{3 E_{g}}\right)\left(e_{4} k_{y} k_{z}+e_{5} k_{x} k_{z}+e_{6} k_{x} k_{y}\right),
\end{array}
$$

where $b$ and $d$ are valence band deformation potentials. Both $b$ and $d$ have wide ranges of reported values and thus uncertainty [52].

From Bahder [47, Eq. 14], $E_{s}$ is $\boldsymbol{k}$-independent and $\pm \sqrt{E_{s}}$ is the contribution to a valence band-edge energy, i.e., $E_{v}(\boldsymbol{k}=0)=-a_{v} \operatorname{tr}([e]) \pm \sqrt{E_{s}}$, with

$$
E_{s}=\frac{b^{2}}{2}\left(\left(e_{1}-e_{2}\right)^{2}+\left(e_{1}-e_{3}\right)^{2}+\left(e_{2}-e_{3}\right)^{2}\right)+d^{2}\left(e_{4}{ }^{2}+e_{5}{ }^{2}+e_{6}{ }^{2}\right),
$$

$E_{s}$ contributes to the splitting of the light and heavy hole bands, but is independent of $\boldsymbol{k}$ and thus does not contribute to DOP. In addition, $E_{s}$ has a decreasing influence as $\boldsymbol{k}$ increases from zero, as can be deduced from Eq. (7) and Sec. 3.3.1. 


\subsubsection{Binomial Expansion}

$E_{v}$, Eq. (7), has a square root dependence on $E_{k}+E_{k s}+E_{s}$. Unless a binomial expansion can be made, the valence band energies are nonlinear in $\boldsymbol{k}^{2}$ and thus difficult to work with.

In the absence of strain $E_{k s}=E_{s}=0$, and $\sqrt{E_{k}}$ gives the difference in masses for the light-hole band and the heavy-hole bands. Given the difference in masses of light and heavy holes and the relatively weak dependence of the light and heavy holes masses on strain, one might expect $E_{k} \gg\left(E_{k s}+E_{s}\right)$ since $E_{k s}$ and $E_{s}$ are strain dependent and $E_{k}$ is strain independent.

Approximate $E_{v}$, Eq. (7), as a binomial series

$$
E_{v} \approx E_{v}^{(0)}+E_{v}^{(1)} \pm\left(\sqrt{E_{k}}+\frac{E_{k s}+E_{s}}{2 \sqrt{E_{k}}}-O\left(\frac{\left(E_{k s}+E_{s}\right)^{2}}{8 E_{k}^{3 / 2}}\right)\right) .
$$

Specialize to $\boldsymbol{k}=\left(0,0, k_{z}\right)$ to simplify the presentation and since determination of the calibration constant $K_{e}$ requires calculation for light polarized parallel to $\boldsymbol{k} ; E_{s}$, Eq. (12), is independent of $\boldsymbol{k}$.

$$
\begin{gathered}
E_{k}\left(0,0, k_{z}\right)=k_{z}{ }^{4}\left(\gamma_{2 m}+\frac{P_{o}{ }^{2}}{3 E_{g}}\right)^{2} \\
E_{k s}\left(0,0, k_{z}\right)=b \times k_{z}{ }^{2} \times\left(\gamma_{2 m}+\frac{P_{o}{ }^{2}}{3 E_{g}}\right)\left(\operatorname{tr}(e)-3 e_{3}\right)
\end{gathered}
$$

Bahder's approximations kept only terms of order $k^{2} e$ [47, pg 1633], thus one should be justified in ignoring the higher-order terms in the binomial expansion of the square root. The neglected term in Eq. (13) is, keeping only terms of lowest order in strain,

$$
O\left(\frac{\left(E_{k s}+E_{s}\right)^{2}}{8 E_{k}^{3 / 2}}\right) \approx 0.22 \frac{\left(e_{1}+e_{2}-2 e_{3}\right)^{2}}{k_{z}^{2}} .
$$

For sufficiently large $k_{z}$ as compared to $e_{1}+e_{2}-2 e_{3}$, the binomial series approximation Eq. (13) converges to Eq. (7). For emission that is $k_{B} T / 2$ above the band edge (for GaAs at room temperature, the band edge emission is at $871 \mathrm{~nm}$, band edge $+k_{B} T / 2=863 \mathrm{~nm}$, or $8 \mathrm{~nm}$ above the band edge), $k_{z}=0.030 \sqrt{\mathrm{eV}}$ for conduction band to light hole transitions and $k_{z}=0.038 \sqrt{\mathrm{eV}}$ for conduction band to heavy hole transitions (for band edge $+k_{B} T, k_{z}>0.043$ ).

The DOP is a sensitive function of strain. DOPs of $\pm 5 \%$ are, for bulk material, extremely large and would be caused by strains of order $0.1 \%$. Take $e_{1}=0.001, e_{2}=0.001, e_{3}=-0.001$, and $k_{z}=0.03$ to find in Eq. (16) that $O()<6 \times 10^{-6}$. Thus one is justified in ignoring the higher order terms in the binomial expansion. Since $E_{s}$ depends on the strain squared, this term can be neglected as compared to terms linear in strain, and the valence band energy for $\boldsymbol{k}=\left(0,0, k_{z}\right)$ can be written as

$$
E_{v}\left(0,0, k_{z}\right) \approx E_{v}^{(0)}\left(0,0, k_{z}\right)+E_{v}^{(1)}\left(0,0, k_{z}\right) \pm\left(k_{z}^{2}\left(\gamma_{2 m}+\frac{P_{o}^{2}}{3 E_{g}}\right)-\frac{b}{2}\left(\operatorname{tr}([e])-3 e_{z z}\right)\right) .
$$

The splitting of light and heavy holes for $\boldsymbol{k}=(0,0, k)$ relative to zero strain is then, to a good approximation,

$$
\Delta=b\left(\operatorname{tr}([e])-3 e_{3}\right),
$$

with one band shifted up by an amount $\Delta / 2$ and the other band shifted down by $\Delta / 2$. A direction-dependent strain shifting of the light hole energies as described by $E_{k s}$, Eq. (11), leads to a DOP for strain.

A similar result to Eq. (17) can be obtained by completing the square in Eq. (13). 


\subsubsection{Free Surface}

Since the surface that is under study in DOP measurements is typically a free surface, this imposes some constraints on the stresses and strains. Stress is force per unit area. $\sigma_{i j} d S$ is the component of force in the $x_{i}$ direction that is transmitted across a surface element of area $\mathrm{d} S$ that is normal to $x_{j}$ [53]. Thus if the ' 2 ' direction is a normal to the free surface, then $\sigma_{22}=0$, $\sigma_{12}=0$ and $\sigma_{32}=0$. Since stress and strain are symmetric second order tensors, $\sigma_{12}=\sigma_{21}$ and $\sigma_{23}=\sigma_{32}$. For a cubic crystal, in Voigt notation, $\sigma_{i}=c_{44} e_{i}, i=4,5,6$. Thus for a cubic crystal, if the shear stress is zero, the shear strain is zero. Since by assumption surface ' 2 ' is a free surface, the normal component of stress $\sigma_{2}=0$ and two of the shear stresses equal zero: $\sigma_{6}=\sigma_{4}=0$. The free surface constraints can be used to simplify the error term in the binomial expansion Eq. (13). In the crystal coordinate system and for a cubic crystal, $c_{11}=c_{22}=c_{33}$, $c_{44}=c_{55}=c_{66}$ and $c_{12}=c_{13}=c_{23}$.

\subsection{Polarized Emissions: $L_{\hat{\boldsymbol{h}}}$ and $L_{\hat{\boldsymbol{v}}}$}

\subsection{1. $\mathrm{DOP}_{[100] \times[001]}$}

Consider $\hat{\boldsymbol{h}}$ along [100], $\hat{\boldsymbol{v}}$ along [001] and $\hat{\boldsymbol{h}} \times \hat{\boldsymbol{v}}$ along [010], and take $x$ along [100], $y$ along [010], and $z$ along [001]. In this section, note that $\hat{\boldsymbol{h}}$ and $\hat{\boldsymbol{v}}$ are definite directions.

Assume that the strain is biaxial, or sufficiently small that the expression $\Delta$, Eq. (18), for the energy shifts with strain of the valence bands are accurate.

Take $\boldsymbol{k}=(0,0, k)$. This gives energy shifts of $\pm b\left(\operatorname{tr}([e])-3 e_{3}\right) / 2$ for the light and heavy holes, relative to zero strain. For $\boldsymbol{k}=(k, 0,0)$ the energy shifts are $\pm b\left(\operatorname{tr}([e])-3 e_{1}\right) / 2$. Note that selection of a direction for $\boldsymbol{k}$ selects different energy shifts, with the direction of strain along the direction of $\boldsymbol{k}$ : one shift depends on $e_{3}$ whereas the other energy shift depends on $e_{1}$. Selection of the polarization for an electromagnetic wave selects the direction for $\boldsymbol{p}$ and hence $\boldsymbol{k}$.

Given the energy shifts $\Delta$, Eq. (18), the density of states (assuming a parabolic band approximation for small $\boldsymbol{k}$ is appropriate) times the probability of occupation is, to within common factors which will cancel in calculation of the DOP, given by

$$
\begin{gathered}
P_{H H}=m_{H H} H^{3 / 2} \exp \left(\frac{+b\left(\operatorname{tr}([e])-3 e_{3}\right)}{2 k_{B} T}\right) \\
P_{L H}=m_{L H}{ }^{3 / 2} \exp \left(\frac{-b\left(\operatorname{tr}([e])-3 e_{3}\right)}{2 k_{B} T}\right) .
\end{gathered}
$$

The irradiance for light polarized in the $z$ direction and produced by carriers with crystal momentum $\boldsymbol{k}=(0,0, k)$ is

$$
\begin{aligned}
L_{\hat{v}}(0,0, k) & =\left|\left\langle C B\left|p_{z}\right| H H_{1}\right\rangle\right|^{2} P_{H H}+\left|\left\langle C B\left|p_{z}\right| L H_{1}\right\rangle\right|^{2} P_{L H} \\
& +\left|\left\langle C B\left|p_{z}\right| H H_{2}\right\rangle\right|^{2} P_{H H}+\left|\left\langle C B\left|p_{z}\right| L H_{2}\right\rangle\right|^{2} P_{L H}
\end{aligned}
$$

where $\langle C B|$ is the Bloch factor (basis function) for one of the conduction bands. See Appendices $\mathrm{B}$ and $\mathrm{C}$. Equation (21) assumes incoherent interaction for luminescence from the conduction band to the the light-hole and heavy-hole bands. Given the broad emission from the band-to-band transitions, this is a reasonable depiction of the interaction for the luminescences from the bands.

After applying the selection rules (see Appendix B) and substituting for $P_{H H}$ and $P_{L H}$

$$
L_{\hat{v}}(0,0, k)=\frac{2|M|^{2}}{3} m_{L H}{ }^{3 / 2} \exp \left(\frac{b\left(\operatorname{tr}([e])-3 e_{3}\right)}{2 k_{B} T}\right) .
$$

A similar procedure gives an expression for the contributions to $L_{\hat{\boldsymbol{h}}}$ :

$$
L_{\hat{\boldsymbol{h}}}(k, 0,0)=\frac{2|M|^{2}}{3} m_{L H}{ }^{3 / 2} \exp \left(\frac{b\left(\operatorname{tr}([e])-3 e_{1}\right)}{2 k_{B} T}\right) .
$$


The total luminescence is the sum over values of $\boldsymbol{k}$, where $\boldsymbol{k}$ gives the crystal momentum for the carriers involved in production of the luminescence. We use the luminescence calculated for $\boldsymbol{k}$ along the two directions of the polarizer as a proxy for the total amount of light that is produced. Under this approximation, $L_{\hat{\boldsymbol{h}}}=L_{\hat{\boldsymbol{h}}}(k, 0,0)$ and $L_{\hat{v}}=L_{\hat{v}}(0,0, k)$.

Under the assumption that the strains are small (typically the magnitude of the strain is $<0.001)$, the exponential functions can be expanded in Taylor series, $\exp (x)=1+x+O\left(x^{2} / 2\right)$, to obtain a simplified expression for the DOP:

$$
\begin{aligned}
\operatorname{DOP}_{\hat{\boldsymbol{h}} \times \hat{\boldsymbol{v}}} & =\frac{L_{\hat{\boldsymbol{h}}}-L_{\hat{\boldsymbol{v}}}}{L_{\hat{\boldsymbol{h}}}+L_{\hat{\boldsymbol{v}}}} \\
& \approx \frac{3 b}{4 k_{B} T}\left(e_{1}-e_{3}\right),
\end{aligned}
$$

with the calibration constant $K_{e}, K_{e}>0$, given by

$$
K_{e}=\frac{-3 b}{4 k_{B} T}=\left|\frac{3 b}{4 k_{B} T}\right|
$$

for $\hat{\boldsymbol{h}}$ along [100] and $\hat{\boldsymbol{v}}$ along [001].

Since $\exp \left(-b 3 e_{3} /\left(2 k_{B} T\right)\right)=1.122$ for $b=-2.0 \mathrm{eV}, k_{B} T=0.026 \mathrm{eV}$, and $e_{3}=0.001$, and $1+b 3 e_{3} /\left(2 k_{B} T\right)=1.115$, and since $\exp \left(b 3 e_{3} /\left(2 k_{B} T\right)\right)=0.8910$, and $1-b 3 e_{3} /\left(2 k_{B} T\right)=$ 0.8846 , differences $<0.72 \%$, clearly the expression for the calibration constant is accurate for strains $\lesssim 0.001$.

The magnitudes of process-induced strains in bulk devices and materials are typically $<0.001$; thus the restriction to small strains to obtain the calibration constant $K_{e}$ is not a significant problem. The approximate expressions for the energy dispersions given by Bahder [47] were obtained by keeping linear terms in the strain (or quadratic if the strain term was contained within a square root). Thus one would expect that expressions derived from Bahder's approximations should be accurate to no more than linear in strain.

Note that the deformation of the sample is, to a very good approximation, linear in the strain fields. One thus uses the principle of linear superposition to find the strain fields from multiple sources. The final strain field, which is the sum of all contributions from all sources, is used to find the DOP of the luminescence. If the final strain is sufficiently small, then the DOP is approximately linear in strain, and the principle of linear superposition holds for the DOP and a calibration constant can be defined. It the strain is not sufficiently small, then it appears that the DOP is not linear in the strain, and one cannot define a calibration constant. However, as a result of linearity in the deformation, small-in-amplitude features will lie on top of larger-in-amplitude features in the DOP and will not be masked by the larger features.

Figure 2 of [2] shows DOP linear over a range $>0.01$. From Table 2 of [7] and the related discussion, the points deviated from linear by no more than 0.6 in 26 when the calibrating force was doubled. For Fig. 3 of [7], a display gain of $10 \times$ was used, which gives just off-scale signals of \pm 2.90 percentage points relative to the green mid-point of the colour bar. The fit of DOP measurements to FEM simulations, as shown in Fig. 3(a) of [7], is remarkably good, which suggests that the DOP is linear over at least $\pm 3 \%$. Assuming the calibration constant $K_{e}=58$, then a DOP or ROP of $3 \%$ corresponds to a strain or strain difference of $5 \times 10^{-4}$; a magnitude of strain which is within the region the small argument approximations, which were made in the derivation of Eq. (26), are accurate.

Typically we use a default display gain of $20 \times$ to display false colour maps of the DOP and ROP. A display gain of $20 \times$ gives just off-scale signals of \pm 1.45 percentage points relative to the green mid-point of the colour bar. Experience has shown that DOPs for bulk material (i.e., not nanostructures) that are off-scale with the default display gains of $20 \times$ indicate areas of concern. 
For a calibration constant of 58, a DOP or ROP of $1.45 \%$ gives a difference of strain or a strain of $0.0145 / 58=2.5 \times 10^{-4}$.

The expression for the calibration constant $K_{e}$ is based on the assumption that the energy splitting for the bands is given by $\Delta$, Eq. (18). For small strains, the strain-squared terms can be ignored and $\Delta$ is accurate for small, but technologically interesting, arbitrary strain distributions

Using the values listed in Appendix A, the calculated calibration constant $K_{e}=58 \pm 30$ for GaAs and InP for $\hat{\boldsymbol{h}}$ along [100] and $\hat{\boldsymbol{v}}$ along [001]. Owing to the cubic symmetry of GaAs and InP, the calibration constant is the same for $\hat{\boldsymbol{h}}$ and $\hat{\boldsymbol{v}}$ equal to any of the equivalent crystal coordinate directions [100], [010], and [001].

Note that $D O P_{\hat{\boldsymbol{h}} \times \hat{v}}$ can be written as $\Delta /\left(2 k_{B} T\right)$ where $\Delta$ is the light hole energy difference for $\boldsymbol{k}$ along the two orthogonal directions $\hat{\boldsymbol{h}}$ and $\hat{\boldsymbol{v}}$ in the measurement plane.

\subsection{2. $k$ not along $z$}

The basis functions (Bloch factors) are defined for $\boldsymbol{k}$ along $z$, with $z$ along a $\langle 001\rangle$ direction in the crystal coordinate system. The basis functions in other directions are obtained by rotation $[48,54]$ of the $|S\rangle,|X\rangle,|Y\rangle,|Z\rangle,|\uparrow\rangle$, and $|\downarrow\rangle$ that are combined to form the conduction band, light hole, heavy hole, and spin orbit states. Momentum matrix elements such as $\varepsilon \cdot\left\langle C B^{\prime}|\boldsymbol{p}| \Psi^{\prime}\right\rangle$ are required to calculate the relative amounts of light in a given polarization where $\left\langle C B^{\prime}\right|$ represents the conduction band, $\left|\Psi^{\prime}\right\rangle$ represents one of the valence band states for a given value of $\boldsymbol{k}$, and $\boldsymbol{\varepsilon}$ is a unit vector that defines the polarization of the luminescence.

Let $R_{t}$ and $S_{t}$ be rotation operators for the spatial and spin components of a wave function. $R_{t}$ and $S_{t}$ commute since they operate on different Hilbert spaces, and $S_{t}$ and $\boldsymbol{p}$ commute since the momentum operator $\boldsymbol{p}$ does not operate on spin. Both rotation operators are unitary. Thus $R_{t} R_{t}^{\dagger}=R_{t}^{\dagger} R_{t}=\boldsymbol{I}$ and $S_{t} S_{t}^{\dagger}=S_{t}^{\dagger} S_{t}=\boldsymbol{I}$ where $\boldsymbol{I}$ is the identity operator.

The matrix elements are obtained by using rotated kets such as $\left|X^{\prime}\right\rangle=R_{t}|X\rangle$ to form the the conduction band, light hole, and heavy hole states. Here $R_{t}$ is a rotation matrix that aligns $z$ with the direction of $\boldsymbol{k}$. These rotated states are used with the components of $\boldsymbol{\varepsilon} \cdot \boldsymbol{p}$ in the crystal coordinate system to calculate the matrix element $M$ for the $\hat{\boldsymbol{h}}$ and $\hat{\boldsymbol{v}}$ polarized transitions. It appears that matrix elements using the rotated states and components of $\boldsymbol{\varepsilon} \cdot \boldsymbol{p}$ in the crystal coordinate system are equal to $\left\langle C B\left|p_{z}\right| \Psi\right\rangle$ with $\langle C B|$ and $|\Psi\rangle$ defined for $\boldsymbol{k}$ along $z$ and in the crystal coordinate system. This simplifies the calculations, particularly since Bahder [47] provided analytic expressions for the energy dispersions of the valence and conduction bands for arbitrary directions of $\boldsymbol{k}$.

Expressions for $\hat{\boldsymbol{h}}$ and $\hat{\boldsymbol{v}}$ not along a crystal coordinate axis are not as compact as for when $\hat{\boldsymbol{h}}$ and $\hat{v}$ are along a direction of high symmetry. We take the limit as $E_{g} \rightarrow \infty$, which reduces Bahder's results from an $8 \times 8$ Hamiltonian to a $4 \times 4$ L-K Hamiltonian [45, Sec. 3] with an insignificant loss of accuracy for the estimation of the calibration constant, to reduce the complexity and space that the typeset equations would otherwise occupy. We found the limit has $\mathrm{a} \approx 2 \%$ effect on $K_{e}$. This loss of accuracy is insignificant as compared to the uncertainty in the material parameters, which are given in Appendix A.

\subsection{3. $\quad R O P<001>$}

The DOP from a (001) surface (i.e., a $z=0$ surface) for an alignment of the $\hat{\boldsymbol{h}}$ (horizontal) polarizer transmission axis at an angle of $\alpha$ with respect to the [100] or $x$-axis can be derived using the procedures described above. Consider two orthogonal directions (e.g., $\hat{\boldsymbol{h}}$ and $\hat{\boldsymbol{v}}$ ) in the $z=0$ plane. Let these two orthogonal directions be defined with respect to a counter-clockwise rotation about the $z$-axis, with the angle $\alpha$ measured with respect to the $x$-axis. The clockwise and counter-clockwise directions are defined for an observer looking at the origin from positive $z$. See Appendix D for more information.

Experimentally, the direction $\hat{\boldsymbol{h}}^{\prime}$ for positive ROP (i.e., $L_{\hat{\boldsymbol{h}}^{\prime}}>L_{\hat{\boldsymbol{v}^{\prime}}}$ ) was chosen to be rotated by 
-45 deg with respect to the direction $\hat{\boldsymbol{h}}$ for positive DOP, (i.e., $L_{\hat{\boldsymbol{h}}}>L_{\hat{\boldsymbol{v}}}$ ) [7, Fig. 1]. This choice leads to a calibration constant for ROP that has the opposite sign as for DOP.

General expressions for $D O P_{001}(\alpha)$ and $R O P_{001}(\alpha)$, in the limit of large $E_{g}$, are

$$
D O P_{001}(\alpha)=\frac{2 \sqrt{3} d \sin (2 \alpha) \gamma_{3} \epsilon_{6}+3 b \cos (2 \alpha) \gamma_{2}\left(\epsilon_{1}-\epsilon_{2}\right)}{2 k_{B} T \sqrt{3 \sin ^{2}(2 \alpha)\left(-\gamma_{2}^{2}+\gamma_{3}^{2}\right)+4 \gamma_{2}^{2}}}
$$

and

$$
R O P_{001}(\alpha)=\frac{-2 \sqrt{3} d \gamma_{3} \epsilon_{6} \cos (2 \alpha)+3 b \sin (2 \alpha) \gamma_{2}\left(\epsilon_{1}-\epsilon_{2}\right)}{2 k_{B} T \sqrt{3 \sin ^{2}(2 \alpha)\left(\gamma_{2}^{2}-\gamma_{3}^{2}\right)+\gamma_{2}^{2}+3 \gamma_{3}^{2}}}
$$

with

$$
\begin{aligned}
& \epsilon_{1}=\cos ^{2}(\alpha) e_{1}+\sin ^{2}(\alpha) e_{2}-\sin (2 \alpha) e_{6} \\
& \epsilon_{2}=\cos ^{2}(\alpha) e_{2}+\sin ^{2}(\alpha) e_{1}+\sin (2 \alpha) e_{6} \\
& \epsilon_{3}=e_{3} \\
& \epsilon_{6}=\cos (2 \alpha) e_{6}+\sin (2 \alpha)\left(\frac{e_{1}-e_{2}}{2}\right)
\end{aligned}
$$

where the $\epsilon_{i}$ are the components of the strain tensor in the crystal coordinate system and the $e_{i}$ are the components of the strain tensor in the DOP measurement coordinate system, which has been rotated by $\alpha$ about [001] (i.e., the $z$-axis). The horizontal direction $\hat{\boldsymbol{h}}$ is taken as the $x^{\prime}$-axis in the rotated (or measurement) coordinate system. The vertical direction $\hat{v}$ is along the axis of rotation, which is the [001] direction, i.e., the $z$-axis or $z^{\prime}$-axis. The same angle $\alpha$ is used for both ROP and DOP; the -45 degree rotation from $\hat{\boldsymbol{h}}$ to $\hat{\boldsymbol{h}}^{\prime}$ has been included in the equation for $R O P_{001}(\alpha)$.

A measurement for $\alpha=0$ gives yields $D O P_{001}(0)=D O P_{[100] \times[010]}(0)=3 b\left(e_{1}-e_{2}\right) /\left(4 k_{B} T\right)$, which gives the calibration constant that was found previously, Eq. (26), for $\hat{\boldsymbol{h}}$ and $\hat{\boldsymbol{v}}$ along crystal coordinate axes.

For measurements from a (001) plane, with $\hat{\boldsymbol{h}}$ parallel to a cleaved (110) facet and $\hat{\boldsymbol{v}}$ perpendicular to the cleaved facet, $\alpha=45 \mathrm{deg}$, giving

$$
D O P_{001}(\alpha=\pi / 4)=\frac{d \gamma_{3}\left(e_{1}-e_{2}\right) \sqrt{3}}{2 k_{B} T \sqrt{\gamma_{2}^{2}+3 \gamma_{3}^{2}}}=K_{e}\left(e_{1}-e_{2}\right) \times \frac{d}{b} \times \frac{2 \gamma_{3}}{\sqrt{3\left(\gamma_{2}^{2}+3 \gamma_{3}^{2}\right)}}
$$

and

$$
R O P_{001}(\alpha=\pi / 4)=-\frac{3 b e_{6}}{2 k_{B} T}=2 K_{e} e_{6} .
$$

This $\alpha=45$ deg example is the case for measurements of a [110] oriented oxide stripe on a (001) growth surface with $\hat{\boldsymbol{h}}$ across the stripe and $\hat{\boldsymbol{v}}$ along the length of the stripe [28, 29,55].

A measurement of $\operatorname{ROP}_{001}(\alpha=\pi / 4)$ gives a measure of the tensor shear strain $e_{6}$ in the measurement system. For an isotropic medium we identified $[3,7]$

$$
R O P_{001}=2 K_{e} e_{6} .
$$

The factor of 2 in the equation arises from recognition that $e_{1}-e_{2}$ in the $\alpha=0$ coordinate system equals $-2 e_{6}$ in the $\alpha=-\pi / 4$ coordinate system.

For GaAs, $\operatorname{ROP}_{001}(\alpha=0)=1.85 e_{6} d /\left(2 k_{B} T\right)$ and the calibration constant equals

$$
K_{e}=\left(\frac{1.85 d}{3 b}\right) \times\left(\frac{-3 b}{4 k_{B} T}\right)
$$


where the expression for $K_{e}$ has been arranged to show the difference between the DOP and the ROP calibration constants. Assuming that $d / b=2.4$, then the calibration constant for $R O P_{001}(\alpha=0)$ is found to be 1.475 times the calibration constant for $D O P_{001}$, rather than the isotropic value of unity times the calibration constant. If $d / b=1.63$, then the calibration constants for $R O P_{001}(\alpha=0)$ and $D O P_{001}(\alpha=0)$ would be equal. For GaAs, Vurgaftman $e t$ al. [52, Table I] report $-1.66<b<-3.9 \mathrm{eV}$ and $-2.7<d<-6.0 \mathrm{eV}$ and recommend $b=-2.0$ $\mathrm{eV}$ and $d=-4.8 \mathrm{eV}$. The ratios for $d / b$ of 1.63 and 2.4 lie within the reported ranges for the values of $b$ and $d$.

Using Bahder's eight-band model, $R O P_{001}(\alpha=0)=1.84 e_{6} d /\left(2 k_{B} T\right)$, which is not significantly different in magnitude than the value of $1.85 e_{6} d /\left(2 k_{B} T\right)$ obtained by taking the limit of large $E_{g}$ to obtain results from a four band model.

\subsection{4. $\quad D O P<110>$}

We wish to calculate DOP and ROP for luminescence from a cleaved facet. A cleaved facet is a free surface and on a free surface the surface tractions must be zero for a body at rest. The ' 2 ' surface has been chosen as the cleavage plane. Thus the components $e_{4}$ and $e_{6}$ are zero, as discussed in Sec. 3.3.2.

Consider three coordinate systems: the crystal coordinate system, a primed system that is rotated about the $z$-axis of the crystal coordinate system by $\alpha$ radians, and a double-primed system that is obtained by rotation of $\beta$ radians about the $y^{\prime}$ axis. See Appendix D and E for details.

Assume strains of $e_{1}, e_{2}, e_{3}$, and $e_{5}$ in the facet coordinate system. For convenience we write $e_{2}$ and do not substitute the value owing to the free surface constraint. The components of the strain tensor $[\epsilon]$ in the crystal coordinate system are, as described in Appendix $\mathrm{F}$, given by $\left[R_{z}(-\alpha)\right] \cdot[e] \cdot\left[R_{z}(-\alpha)\right]^{T}$ (or, depending on one's perspective, $\left.\left[R_{z}(\alpha)\right]^{T} \cdot[e] \cdot\left[R_{z}(\alpha)\right]\right)$ and are equal to

$$
[\epsilon]=\left[\begin{array}{ccc}
e_{1} \cos ^{2}(\alpha)+e_{2} \sin ^{2}(\alpha) & \sin (2 \alpha)\left(e_{1}-e_{2}\right) / 2 & \cos (\alpha) e_{5} \\
\sin (2 \alpha)\left(e_{1}-e_{2}\right) / 2 & e_{1} \sin ^{2}(\alpha)+e_{2} \cos ^{2}(\alpha) & \sin (\alpha) e_{5} \\
\cos (\alpha) e_{5} & \sin (\alpha) e_{5} & e_{3}
\end{array}\right]
$$

The components of the strain tensor in the crystal coordinate system $\epsilon_{i}$ are used in the expressions for $E_{v}$ to obtain the strain-induced splitting of the valence band.

We are interested in the calibration constant for a cleaved facet, thus we are interested in the case $\alpha= \pm \pi / 4$. The rotation of $\beta$ about the $y^{\prime}$ axis is used to align $z^{\prime \prime}$ along any direction in the plane of the facet. The rotation of $\beta$ about the $y^{\prime}$ axis will be needed for a calculation of the DOP for an arbitrary direction in the plane of the facet and to calculate the ROP. We calculate the DOP for $\boldsymbol{k}_{\boldsymbol{x}^{\prime}}=(k, 0,0)$ and $\boldsymbol{k}_{z^{\prime}}=(0,0, k)$ in the $x^{\prime} y^{\prime} z^{\prime}$ facet coordinate system, which means that $\beta=0$. Assuming that $\boldsymbol{k}_{\boldsymbol{x}^{\prime}}$ is parallel to a growth plane, then $\beta=0$ would correspond to the usual alignment for a measurement of DOP (see Fig. 1 of [7]). The components of $\boldsymbol{k}_{\boldsymbol{x}^{\prime}}$ and $\boldsymbol{k}_{z^{\prime}}$ in the crystal coordinate system are given by $\left[R_{z}(\alpha)\right]^{T} \cdot(k, 0,0)^{T}$ and $\left[R_{z}(\alpha)\right]^{T} \cdot(0,0, k)^{T}$ where $\left[R_{z}(\alpha)\right]$ is the rotation matrix for the coordinate transformation, which is a rotation about the $z$-axis by $\alpha$ radians. See Appendices D and E.

The calculation for the rate of luminescence for carriers with $\boldsymbol{k}_{z^{\prime}}=\boldsymbol{k}_{z}$ proceeds as detailed previously, as the rotation does not affect the $z$-direction. The calculation for $\boldsymbol{k}_{\boldsymbol{x}^{\prime}}$ proceeds slightly differently as now the crystal momentum has components in both the $x$ and $y$ directions. In the crystal coordinate system, $\boldsymbol{k}_{\boldsymbol{x}^{\prime}}=(k \cos (\alpha), k \sin (\alpha), 0)$ and $\boldsymbol{k}_{z^{\prime}}=\boldsymbol{k}_{z}=(0,0, k)$ where $k$ is the 
magnitude of the crystal momentum. From Eq. (11),

$$
\begin{array}{r}
\lim _{E_{g} \rightarrow \infty} E_{k s}(\cos (\alpha), \sin (\alpha), 0)=b \gamma_{2}\left(\operatorname{tr}([\epsilon])-3 \cos ^{2}(\alpha) \epsilon_{1}-3 \sin ^{2}(\alpha) \epsilon_{2}\right) \\
-\sqrt{3} d \gamma_{3} \sin (2 \alpha) \epsilon_{6} .
\end{array}
$$

Two of the shear terms in Eq. (11) are zero because $k_{z}=0$. The remaining shear term is antisymmetric in $\alpha$.

The energy difference for the DOP in the plane of the facet, $\Delta_{f}(\beta)=\Delta(\alpha=\pi / 4, \beta)$, with $\beta=0$ is, from Eq. (11),

$$
\begin{aligned}
\Delta_{f}(\beta=0) & =+\frac{\left(d \gamma_{3} \sqrt{3}+3 b \gamma_{2}\right) e_{1}}{2 \mathcal{D}}+\frac{\left(-d \gamma_{3} \sqrt{3}+3 b \gamma_{2 L}\right) e_{2}}{2 \mathcal{D}} \\
& -\frac{3 b e_{3}}{2}+\frac{\left(\mathcal{D}-2 \gamma_{2}\right) \operatorname{tr}([e]) b}{2 \mathcal{D}}
\end{aligned}
$$

where the denominator $\mathcal{D}=\sqrt{\gamma_{2}^{2}+3 \gamma_{3}^{2}}$.

$e_{2}$ in the expression for $\Delta_{f}(\beta=0)$ can be eliminated by substitution of the free surface condition to obtain

$$
\begin{aligned}
\Delta_{f}(\beta=0) & = \\
& -\frac{\left(-d \gamma_{3}\left(c_{12}^{\prime} / c_{22}^{\prime}+1\right) \sqrt{3}+\left(c_{12}^{\prime} / c_{22}^{\prime}-1\right)\left(\mathcal{D}+\gamma_{2}\right) b\right) e_{1}}{2 \mathcal{D}} \\
& -\frac{\left(d \gamma_{3 L} c_{23}^{\prime} / c_{22}^{\prime} \sqrt{3}+\left(c_{23}^{\prime} / c_{22}^{\prime}+2\right)\left(\mathcal{D}+\gamma_{2}\right) b\right) e_{3}}{2 \mathcal{D}} .
\end{aligned}
$$

For GaAs, with $d=2.4 b, \Delta_{f}(\beta=0)=1.88 b e_{1}-1.22 b e_{3}$. For $d=1.485 b, \Delta_{f}(\beta=0)=$ $2.75 b\left(e_{1}-e_{3}\right) / 2$.

If $\Delta_{f}(\beta=0)=3 b\left(e_{1}-e_{3}\right) / 2$, then the calibration constant for DOP measurements with $\beta=0$ on cleaved facets (i.e., $\{110\}$ surfaces) would be identical to the calibration constant for DOP measurements on $\{001\}$ surfaces with $\alpha=0$. Given the calculations described here, this would require $d=1.485 b$ to obtain an $\left(e_{1}-e_{3}\right)$ dependence but the magnitude of the calibration constants for $\{001\}$ and $\{110\}$ surfaces would not be the same.

The axes for a calculation of ROP in the plane of the facet $(\alpha=\pi / 4)$ and in the usual alignment (see Fig. 1 of [7]) are obtained for $\beta= \pm 45$ deg. Following the steps for a calculation of $R O P_{1 \overline{1} 0}$, one should find

$$
\Delta_{f}(\beta=\pi / 4)=-\frac{4 \sqrt{3} e_{5} d \gamma_{3}}{\sqrt{\gamma_{2}^{2}+15 \gamma_{3}^{2}}}
$$

which, for GaAs, equals $=-1.76 e_{5} d$. ROP is, as expected, proportional to the shear strain $e_{5}$ in the plane of the facet. For $R O P_{001}, \Delta(\alpha=0)=-1.85 e_{6} d$, which means that the calculated $R O P_{001}$ and $R O P_{1 \overline{1} 0}$ calibration constants differ by about 1 part in 20 . However, the recommended value of $d=-4.8 \mathrm{eV}$ gives a calibration constant that appears to be about $1.6 \times$ larger than the value that is expected, based on experimental measurements reported in [7].

The expression for $D O P_{1 \overline{1} 0}$ is more complicated than the expressions for ROP or $D O P_{\langle 001\rangle}$; this owes to lack of symmetry as compared to other cases. For $R O P_{1 \overline{1} 0}$, the two $\boldsymbol{k}$ 's are in the plane of the facet and are mirror images along the [001] axis. These two directions are equivalent crystal directions. For $D O P_{110}$, the two directions for $\boldsymbol{k}$ are along [110] and along [001]. These directions are not equivalent directions in the crystal. 


\section{Discussion}

Results presented in this paper suggest that for InP, $D O P_{1 \overline{0} 0}=\left(1.32 e_{1}-0.80 e_{3}\right) b /\left(4 k_{B} T\right)$, where $d=2.5 b$, which is the recommended ratio [52], has been used to eliminate $d$ from the expression.

The calibration constant for $\mathrm{InP}$ was determined by fits of finite element method simulations to $\mathrm{V}$ grooves that were placed in a bending moment [7]. Least square fits of $-C_{e}\left(e_{1}-e_{3}\right)$ were found to fit better to the data than fits of $-C_{\sigma}\left(\sigma_{1}-\sigma_{3}\right)$, where $[\sigma]=[c][e]$ is the stress tensor and $[\mathrm{c}]$ is the stiffness. This is surprising given that

$$
\begin{aligned}
\sigma_{1}-\sigma_{3} & = \\
& +\left(c_{11}-c_{13}-\frac{\left(c_{12}-c_{23}\right) c_{12}}{c_{22}}\right) e_{1}-\left(\frac{\left(c_{12}-c_{23}\right) c_{23}}{c_{22}}+\left(c_{13}-c_{33}\right)\right) e_{3} \\
& =\left(72.9 e_{1}-34.8 e_{3}\right) \times 10^{9}
\end{aligned}
$$

where numerical values for a $\{110\}$ facet of InP have been substituted. Both the difference of stress and the theoretical expression for $D O P_{\langle 110\rangle}$ have 'excess' $e_{1}$. One would not necessarily expect a good fit of $e_{1}-e_{3}$ to the data for a situation with excess $e_{1}$, particularly given that fits of simulations using isotropic material properties did not fit as well as orthotropic simulations. These results suggest that the values of $b$ and $d$ are not accurate, or that the analysis is inaccurate.

Vurgaftman et al. [52, pg 5824] write

"The greatest uncertainty in the GaAs band structure parameters is associated with the deformation potentials,..."

"The various values for the deformation potential $b$ varied between -1.66 and -3.9 $\mathrm{eV}$, although recent results tend toward the lower end of that range. We propose the following composite values [for GaAs]: $b=-2.0 \mathrm{eV}$ and $d=-4.8 \mathrm{eV}$, which are consistent with the vast majority of measurements and several calculations."

The calibration constant is a function of the deformation potential $b$; a wide range of uncertainty in the value of $b$ implies a wide range in the value of the calibration constant $K_{e}$. Vurgaftman $e t$ al. [52, pg 5829] in Table VI give the range of $b$ for InP as [-1.0,-2.0]. In Table I of Ref. [52, pg 5825], the range of $b$ for GaAs is listed as [-1.66, -3.9].

In addition, it was found that the best fit ROP calibration constant was found to be $1.3 \times$ larger than expected [7, pg 1817]. The factor of $1.3 \times$ was explained at the time as owing to an uneven mounting surface providing an additional bending moment to the sample. The calculated expression for InP is $R O P\langle 110\rangle=-1.76 d e_{5} /\left(2 k_{B} T\right)$. Using the recommended value of $d=-5.0$ for InP gives $R O P_{\langle 110\rangle}=2 \times 85 e_{5}$, which is $1.31 \times$ larger than the expected $2 \times 65 e_{5}$. Given the results obtained, the assumption that the calibration constants for ROP and DOP are equal needs to be re-examined, as does the explanation for the difference in the constants found by fitting.

\subsection{Wishful Thinking on $\boldsymbol{b}$ and $\boldsymbol{d}$ ? Invariants of Second Order Tensors}

There are three principal invariants for a second rank tensor. These invariants are the same in any coordinate system obtained by rotations about the original coordinate system. The trace of a tensor is a principal invariant. For the strain tensor $[e]$ in Voigt notation, Eq. (1), the second principal invariant $I_{2}([e])$ is

$$
I_{2}([e])=e_{1} e_{2}+e_{2} e_{3}+e_{1} e_{3}+e_{4}^{2}+e_{5}^{2}+e_{6}^{2} .
$$

The third principal invariant of a second rank tensor is the determinant of the tensor. 
Consider the expression for $I_{2}([e-[I] \operatorname{tr}([e]) / 3])$ when the hydrostatic component of strain, which equals one third of the trace of the strain tensor, has been subtracted off of the diagonal elements of the strain tensor $[e]$. Here $[\boldsymbol{I}]$ is the identity matrix. For this case

$$
I_{2}([e-[\boldsymbol{I}] \operatorname{tr}([e]) / 3])=\frac{\left(e_{1}-e_{2}\right)^{2}}{6}+\frac{\left(e_{2}-e_{3}\right)^{2}}{6}+\frac{\left(e_{1}-e_{3}\right)^{2}}{6}+e_{4}{ }^{2}+e_{5}{ }^{2}+e_{6}{ }^{2} .
$$

$I_{2}([e-[\boldsymbol{I}] \operatorname{tr}([e]) / 3])$ is a von Mise strain.

Compare the expression for $I_{2}([e-[\boldsymbol{I}] \operatorname{tr}([e]) / 3])$ to Bahder's [47] expression for the strain-only contribution Eq. (12) to the valence band energy (7) and note that

$$
\left.E_{s}\right|_{d=\sqrt{3} b}=-3 b^{2} I_{2}([e-[\boldsymbol{I}] \operatorname{tr}([e]) / 3]) .
$$

If $d=\sqrt{3} b$ then the strain-only contribution to the valence band energy, $E_{S}$, is invariant of the coordinate system.

\subsubsection{Isotropic Material}

For an isotropic material, $\gamma_{2}=\gamma_{3}$, and there are only two independent elastic constants: $c_{11}=c_{22}=c_{33}, c_{12}=c_{23}=c_{13}$ with $c_{44}=c_{55}=c_{66}=\left(c_{11}-c_{12}\right) / 2$. Of the Luttinger parameters, $\gamma_{1}$ and $\gamma_{2}$ describe the coupling of the valence band basis states $|X\rangle,|Y\rangle$, and $|Z\rangle$ to other states, and $\gamma_{3}-\gamma_{2}$ is a measure of the anisotropy of the band structure.

Assuming $\gamma_{3}=\gamma_{2}, d=\sqrt{3} b$, and using isotropic elastic constants,

$$
D O P_{1 \overline{1} 0}=\frac{3 b}{4 k_{B} T}\left(e_{1}-e_{3}\right)=-K_{e}\left(e_{1}-e_{3}\right),
$$

which is as expected, with $K_{e}$ given by Eq. (26). For an isotropic material, the calculated DOP is proportional to the difference of the normal components of strain that are along the two orthogonal transmission axes of the polarizer, provided that $d=\sqrt{3} b$. For the isotropic case, the calculated $R O P=-3 b /\left(2 k_{B} T\right) e_{5}=2 K_{e} e_{5}$, which also is as expected, provided that $d=\sqrt{3} b$; the isotropic ROP equals the bilinear product of $2 K_{e}$ and the shear strain in the plane of the measured surface. These isotropic results are obtained if and only if the shear deformation potential $d=\sqrt{3} b$.

\subsubsection{GaAs and InP: Cubic Materials}

The calculated values of the DOP are closer to results obtained experimentally [7] if it is assumed that $d=\sqrt{3} b$. For this assumption and for GaAs,

$$
D O P_{1 \overline{1} 0}=\left(1.0 e_{1}-0.89 e_{3}\right) \frac{b}{4 k_{B} T}
$$

by substitution of GaAs values into Eq. (40). For InP, the result is similar: $D O P_{1 \overline{1} 0}=$ $\left(1.01 e_{1}-0.91 e_{3}\right) b /\left(4 k_{B} T\right)$. For both InP and GaAs, the 'excess' $e_{1}$ is substantially reduced if $d=\sqrt{3} b$. For InP with $d=\sqrt{3} b$, the ratio of the $e_{1}$ coefficient to the $e_{3}$ coefficient is 1.12 , whereas for the recommended ratio of $d=2.5 b$, the ratio of the coefficients is 1.63 . This first result with $d=\sqrt{3} b$ is consistent with experimental results [7] for the DOP calibration constant, where the trial function had no excess $e_{1}$ and appeared to fit the data well. For an isotropic material or for both $\hat{\boldsymbol{h}}$ and $\hat{\boldsymbol{v}}$ along crystal coordinate directions, $D O P=3 b \times\left(e_{1}-e_{3}\right) /\left(4 k_{B} T\right)$.

From Eq. (41) with $d=\sqrt{3} b$ and for GaAs,

$$
R O P_{1 \overline{1} 0}=-1.02 \frac{3 b}{4 k_{B} T} \times 2 e_{5},
$$


with the same numerical result for InP to two decimal places. The calculated calibration constant with $d=\sqrt{3} b$ for $R O P_{110}$ in GaAs is $1.02 \times$ greater than for the isotropic case or for a $\{010\}$ plane. Experimentally the $R O P_{1 \overline{1} 0}$ calibration constant was found to be $1.3 \pm 0.1$ times greater than the $D O P_{1 \overline{1} 0}$ calibration constant for InP [7].

With $d=\sqrt{3} b$, one finds that the calculated DOP appears to be closer to the experimentally determined DOP, whereas the calculated ROP appears to be further from the experimental result. The fit to the ROP data showed an asymmetry (see Fig. 3(b) of [7]) which was used to suggest that an additional bending moment was added by the platform that supported the chip and that this explained the finding of a ROP calibration constant that was $(1.3 \pm 0.1) \times$ the DOP calibration constant.

Arbitrarily setting $d=\sqrt{3} b$ does not solve all discrepancies between data and calculation. The results do suggest that it might be worthwhile to investigate further the relationship, if any, between the deformation potentials.

The ratio of the experimentally determined calibration constants for GaAs and InP was found to be $(50 \pm 10):(65 \pm 10)$ [9]. Since the calibration constant is a linear function of the deformation potential $b$ and $b$ is dominant in determining the calibration constant, this suggests that the ratio of the strain deformation potentials for GaAs and InP should be roughly the same as the ratio of the calibration constants. Thus one might expect $b_{\mathrm{InP}}=(1.3 \pm 0.3) \times b_{\mathrm{GaAs}}$.

\section{Conclusion}

Analysis of the degree of polarization (DOP) of luminescence is a useful and sensitive technique to estimate strain in luminescent IIII-V materials and devices. Previously we assumed that $D O P=-K_{e}\left(e_{1}-e_{2}\right)$ and determined experimentally the calibration constant for GaAs and InP, where $e_{1}$ and $e_{2}$ are normal strains near the surface being measured. The rotated degree of polarization, or ROP, can be measured by rotating the transmission axes of the polarizer by -45 deg relative to the settings for a DOP measurement. The ROP is a measure of the shear strain $e_{12}$, with assumed form $R O P=2 K_{e} e_{12}$. The calibration constant $K_{e}$ was previously measured experimentally and the assumed dependence of the DOP and ROP on strain were found to be consistent with experimental data.

A simplified analytic determination of the dependence of DOP and ROP on strain is presented here and discussed. This simplified analysis gives expressions for the calibration constant for InP and GaAs, and for different surfaces. Analytic expressions for the band energies with arbitrary crystal momentum, which were derived and published by T. B. Bahder [45-47], were used with Boltzmann statistics, effective density of states, rotation of the band-edge Bloch factors (basis functions), and selection rules for given polarizations of light, to obtain transitions rates and hence the DOP and the ROP. To avoid elliptic integrals, the light along the two orthogonal directions for the polarizer that define the ' 1 ' and ' 2 ' directions were used as proxies for the total amount of light emitted in the selected polarization. The calibration constant $K_{e}$ was found for technologically interesting strains to be linearly dependent on the deformation potentials $b, d$, or on both $b$ and $d$, depending on the surface and the orientation of the polarizer transmission axes relative to the crystal coordinate system.

The previously assumed forms for the DOP and ROP were found to be consistent with an isotropic material, provided that the deformation potentials $b$ and $d$ were linearly related with $d=\sqrt{3} b$. If $d \neq \sqrt{3} b$, then the DOP and ROP were not isotropic even though the material was assumed to be isotropic. This fact raises some interesting questions.

Experimentally, the calibration constant $K_{e}$ was measured on cleaved facets to be $65 \pm 10$ for InP [7,9] and $50 \pm 10$ for GaAs [2,3,9]. These experimentally measured values for $K_{e}$ are consistent with the theoretically determined values for the calibration constant, given the large range in the reported values for the deformation potentials $b$ and $d$ [52]. The calculated relationship between DOP and strain for a (110) facet was found to be closer to experimental 
results if it was assumed that $d=\sqrt{3} b$. Given the difference between the calibration constant for GaAs and InP, it appears that $b_{\mathrm{InP}}>b_{\mathrm{GaAs}}$, and based on the ratios of the calibration constants, $b_{\text {InP }} \approx 1.3 \times b_{\text {GaAs }}$.

Further work on determination of the calibration constant $K_{e}$ and the relationship, if any, between $b$ and $d$ is indicated. The degree of polarization of luminescence in InP and GaAs is a sensitive function of strain. Careful measurements of the dependence of DOP and ROP on strain might lead to better values for and understanding of the deformation potentials $b$ and $d$.

\section{Disclosures}

The authors declare no conflict of interest.

\section{References}

1. D. T. Cassidy and C. S. Adams, "Polarization of the output of InGaAsP semiconductor diode lasers," IEEE J. Quantum Electron. 24, 1156-1160 (1989).

2. P. D. Colbourne and D. T. Cassidy, "Bonding stress measurements from the degree of polarization of facet emission of AlGaAs superluminescent diodes," IEEE J. Quantum Electron. 27, 914-920 (1991).

3. P. D. Colbourne and D. T. Cassidy, "Observation of dislocation stresses in InP using polarization-resolved photoluminescence,” Appl. Phys. Lett. 61, 1174-1176 (1992).

4. P. D. Colbourne and D. T. Cassidy, "Imaging of stresses in GaAs diode lasers using polarization resolved photoluminescence," IEEE J. Quantum Electron. 29, 62-68 (1993).

5. P. D. Colbourne and D. T. Cassidy, "Dislocation detection using polarization-resolved photoluminescence," Can. J. Phys. 70, 803-812 (1992).

6. C. S. Adams and D. T. Cassidy, "Effects of stress on threshold, wavelength and polarization of the output of InGaAsP semiconductor diode lasers," J. Appl. Phys. 64, 6631-6638 (1988).

7. D. T. Cassidy, S. K. K. Lam, B. Lakshmi, and D. M. Bruce, "Strain mapping by measurement of the degree of photoluminescence," Appl. Opt. 43, 1811-1818 (2004).

8. D. T. Cassidy, "Spatially resolved and polarization resolved photoluminescence for study of strain and dislocations in III-V materials," Mater. Sci. Eng. B 91-92, 2-9 (2002)

9. J.-P. Landesman, D. T. Cassidy, J. W. Tomm, and M. L. Biermann, "Strain measurement," in Quantum-Well Laser Array Packaging: Nanoscale Packaging Techniques, J. W. Tomm and J. Jiménez, eds. (McGraw-Hill Companies, New York, 2007).

10. D. T. Cassidy, C. K. Hall, O. Rehioui, and L. Béchou, "Strain estimation in III-V materials by analysis of the degree of polarization of luminescence," Microelectron. Reliab. 50, 462-466 (2010).

1. M. A. Fritz and D. T. Cassidy, "Cooling rate in diode laser bonding," IEEE Trans. Components Packag. Technol. 27, 147-154 (2004).

2. M. A. Fritz and D. T. Cassidy, "Extraction of bonding strain data in diode lasers from polarzation-resovled photoluminescence measurements," Microelectron. Reliab. 44, 787-796 (2004).

3. D. T. Cassidy, "Rotation of principal axes and birefringence in III-V lasers owing to bonding strain," Appl. Opt. 52, 6258-6265 (2013).

14. M. L. Biermann, D. T. Cassidy, T. Q. Tien, and J. W. Tomm, "Processing induced strains at solder interfaces in extended semiconductor structures," J. Appl. Phys. 101, 114512 (2007).

15. F. H. Peters and D. T. Cassidy, "Scattering, absorption, and anomalous spectral tuning of $1.3 \mu \mathrm{m}$ semiconductor diode lasers," J. Appl. Phys. 71, 4140-4144 (1992).

6. J. Yang and D. T. Cassidy, "Strain measurement and estimation of photoelastic effects and strain-induced gain change in ridge waveguide lasers," J. Appl. Phys. 77, 3382-3387 (1995).

17. M. A. Fritz and D. T. Cassidy, "Photoelastic effect from die bonding of diode lasers," Appl. Opt. 43, 160-166 (2004).

18. M. G. Daly, D. M. Bruce, P. E. Jessop, D. T. Cassidy, and D. Yevick, "Metallization stress in weakly guiding InP/InGaAsP waveguides," Semicond. Sci. Technol. 9, 1387-1390 (1994).

19. D. Lisak, D. T. Cassidy, and A. H. Moore, "Bonding stress and reliability of high-power GaAs-based lasers," IEEE Trans. Components Packag. Technol. 24, 92-98 (2001).

20. M. A. Fritz, G. B. Morrison, and D. T. Cassidy, "The effect of die bonding distributed feedback lasers," IEEE J. Quantum Electron. 40, 996-1002 (2004).

21. G. B. Morrison, D. T. Cassidy, J. E. Johnson, M. Sipics, H. Wang, and R. Wolf, "Bonding-induced strain effects in InP DFB components soldered p-side-up on AIN substrates," IEEE J. Quantum Electron. 45, 937-944 (2009).

22. A. Gupta, G. C. Weatherly, D. T. Cassidy, and D. M. Bruce, "Characterization and modelling of the strain fields associated with InGaAs layers on V-grooved InP substrates,” J. Appl. Phys. 82, 6016-6023 (1997).

23. A. Borowiec, D. M. Bruce, D. T. Cassidy, and H. K. Haugen, "Imaging strain fields resulting from laser micromachining of semiconductors," Appl. Phys. Lett. 83, 225-227 (2003).

24. S. Saha, D. T. Cassidy, and D. A. Thompson, "Investigation of cross-hatch in $\operatorname{In}_{0.3} \mathrm{Ga}_{0.7}$ As pseudo-substrates," J. Appl. Phys. 113, 124301 (2013). 
25. S. Saha, D. T. Cassidy, and D. A. Thompson, "Comparison of different grading approaches in metamorphic buffers grown on GaAs substrates," J. Cryst. Growth 386, 183-189 (2014).

26. D. T. Cassidy, O. Rehioui, C. K. Hall, L. Béchou, Y. Deshayes, A. Kohl, T. Fillardet, and Y. Ousten, "High-power laser bars and shear strain," Opt. Lett. 38, 1633-1635 (2013).

27. M. Mokhtari, P. Pagnod-Rossiaux, F. Laruelle, J.-P. Landesman, A. Moréac, C. Levallois, and D. T. Cassidy, "Optical characterizations of VCSEL for emission at $850 \mathrm{~nm}$ with Al oxide confinement layers," J. Electron. Matls. 47, 4987-4992 (2018).

28. J.-P. Landesman, D. T. Cassidy, M. Fouchier, E. Pargon, C. Levallois, M. Mokhtari, J. Jiménez, and A. Torres, "Mechanical stress in InP structures etched in an inductively coupled plasma reactor with $\mathrm{Ar} / \mathrm{Cl}_{2} / \mathrm{CH}_{4} \mathrm{plasma}$ chemistry," J. Electron. Matls. 47, 4964-4969 (2018).

29. J.-P. Landesman, D. T. Cassidy, M. Fouchier, C. Levallois, E. Paragon, N. Rochat, M. Mokhtari, J. Jiménez, and A. Torres, "Mapping of mechanical strain induced by thin and narrow dielectric stripes on InP surfaces," Opt. Lett. 43, 3505-3508 (2018).

30. Z.-H. Yan and S. Zhou, "Bonding stress and reliability of low-polarization quantum-well superluminescent diode," Phys. E: Low-dimensional Syst. Nanostructures 109, 140-143 (2019).

31. M. Fouchier, N. Rochat, E. Pargon, and J.-P. Landesman, "Polarized cathodoluminescence for strain measurement," Rev. Sci. Instrumen. 90, 043701 (2019).

32. J. Chen, J. Zhang, J. Dai, F. Wu, S. Wang, H. Long, R. Liang, J. Xu, C. Chen, Z. Tang, Y. He, M. Li, and Z. Feng, "Strain dependent anisotropy in photoluminescence of heteroepitaxial nonpolar $a$-plane ZnO layers," Opt. Mater. Express 7, 3944-3951 (2017).

33. T.-W. Lin, L. P. Rowe, A. J. Kaczkowski, G. P. Horn, and H. T. Johnson, "Polarization-resolved imaging for both photoelastic and photoluminescence charcaterization of silicon wafers," Exp. Mech. 56, 1339-1350 (2016).

34. M. P. Peloso, L. Meng, and C. S. Bhatia, "Combined thermography and luminescence imaging to characterize the spatial permformance of multicrystalline Si wafer solar cells," IEEE J. Quantum Electron. 5, 102-111 (2015).

35. M. Winterfeldt, P. Crump, H. Wenzel, G. Erbert, and G. Tranäkle, "Experimental investigation of factors limiting slow axis beam quality in 9xx nm high power broad area diode lasers," J. Appl. Phys. 116, 063102 (2014).

36. M. Hempel, M. Ziegler, S. Schwirzke-Schaaf, J. W. Tomm, D. Jankowski, and D. Schroeder, "Spectroscopic analysis of packaging concepts for high-power diode laser bars," Appl. Phys. A - Mater. Sci. Process. 107, 371-377 (2012)

37. Y. Matsui, D. Vakhshoori, P. D. Wang, P. L. Chen, C. C. Lu, M. Jiang, K. Knopp, S. Burroughs, and P. Tayebati, "Complete polarization mode control of long-wavelength tunable vertical-cavity-surface-emitting-lasers over 65-nm tuning, up to $14 \mathrm{~mW}$ output power,” IEEE J. Quantum Electron. 39, 1037-1048 (2003).

38. W. Pittroff, G. Erbert, G. Beister, F. Bugge, A. Klein, A. Knauer, J. Maege, P. Ressel, J. Sebastian, R. Staske, and G. Traenkle, "Mounting of high power laser diodes on boron nitride heat sinks using an optimized Au/Sn metallurgy," IEEE Trans. Adv. Pack. 24, 434-441 (2001).

39. A. Gustafsson, M.-E. Pistol, L. Montelius, and L. Samuelson, "Local probe techniques for luminescence studies of low-dimensional semiconductor structures," J. Appl. Phys. 84, 1715-1775 (1998).

40. J. W. Tomm, T. Q. Tien, and D. T. Cassidy, "Spectroscopic strain measurement methodology: Degree-of-polarization photoluminescence versus photocurrent spectroscopy,” Appl. Phys. Lett. 88, 133504 (2006).

41. B. Lakshmi, D. T. Cassidy, and B. J. Robinson, "Quantum-well strain and thickness characterization by degree of polarization,” J. Appl. Phys. 79, 7640-7645 (1996).

42. B. Lakshmi, B. J. Robinson, D. T. Cassidy, and D. A. Thompson, "Anisotropic interfacial strain in InP/InGaAs/InP quantum wells studied using degree of polarization of photoluminescence,” J. Appl. Phys. 81, 3616-3620 (1997).

43. B. Lakshmi, D. T. Cassidy, and B. J. Robinson, “Anisotropic interfacial strain in InP/InGaAs/InP quantum wells,” J. Appl. Phys. 84, 5739-5742 (1998).

44. S. J. Lascos and D. T. Cassidy, "Optical phase and intensity modulation from a rotating optical flat: effect on noise in degree of polarization measurements," Appl. Opt. 48, 1697-1704 (2009).

45. T. B. Bahder, "Eight-band $\boldsymbol{k} \cdot \boldsymbol{p}$ model of strained zinc-blende crystals," Phys. Rev. B 41, 11992-12001 (1990).

46. T. B. Bahder, "Erratum: Eight-band $\boldsymbol{k} \cdot \boldsymbol{p}$ model of strained zinc-blend crystals [Phys. Rev. B 41, 11992 (1990)]," Phys. Rev. B 46, 9913 (1992).

47. T. B. Bahder, "Analytic disperson relations near the $\Gamma$ point in strained zinc-blende crystals," Phys. Rev. B $\mathbf{4 5}$, 1629-1637 (1992).

48. P. Enders, "A new approximation for block diagonalizing the eight-band $\boldsymbol{k} \cdot \boldsymbol{p}$ Hamiltonian," Phys. Stat. Sol. (b) 187, 541-550 (1995).

49. T. E. Ostromek, "Evaluation of the matrix elements of the $8 \times 8 \boldsymbol{k} \cdot \boldsymbol{p}$ Hamiltonian with $\boldsymbol{k}$-dependent spin-orbit contributions for the zinc-blende structure of GaAs," Phys. Rev. B 54, 14467-14479 (1996).

50. K. M. van Vliet and A. H. Marshak, "The effective density of states in the conduction and valence bands for arbitrary band structure," Phys. Stat. Sol. (b) 101, 525-530 (1980).

51. C. Y.-P. Chao and S. L. Chuang, "Spin-orbit-coupling effects on the valence-band structure of strained semiconductor quantum wells," Phys. Rev. B 46, 4110-4122 (1992).

52. I. Vurgaftman, J. R. Meyer, and L. R. Ram-Mohan, "Band parameters for III-V compound semiconductors and their alloys," J. Appl. Phys. 89, 5815-5875 (2001).

53. J. F. Nye, Physical Properties of Crystals (Oxford Unversity Press, New York, 1985).

54. E. O. Kane, "Band structure of indium antimonide,” J. Phys. Chem. Solids. 1, 249-261 (1957). 
55. S. Gérard, M. Mokhtari, J.-P. Landesman, C. Levallois, M. Fouchier, E. Pargon, P. Pagnod-Rossiaux, F. Laruelle, A. Moréac, B. Ahammou, and D. T. Cassidy, "Photoluminescence mapping of the strain induced in InP and GaAs substrates by $\mathrm{SiN}_{x}$ stripes etched from thin films grown under controlled mechanical stress," Thin Solid Films $\mathbf{x x}$, xxxx (2020).

56. S. Gasiorowicz, Quantum Physics (John-Wiley, 1974).

57. G. Lasher and B. Stern, "Spontaneous and stimulated emission in semiconductors," Phys. Rev. 133, A553-A563 (1964).

58. G. Baym, Lectures on Quantum Mechanics (W. A. Benjamin, Inc., Reading MA, USA, 1978).

59. P. W. Milonni and J. H. Eberly, Lasers (John Wiley and Sons, New York, 1988).

60. F. W. Byron and R. W. Fuller, Mathematics of Classical and Quantum Physics (Dover Books on Physics, 1992), revised ed. 
A. Physical and Material Values

Table 1. Physical constants.

\begin{tabular}{ccccc}
\hline parameter & symbol & value & unit & remarks \\
\hline electron mass & $m_{o}$ & $9.10938356 \times 10^{-31}$ & $\mathrm{~kg}$ & \\
electron charge & $q$ & $1.6021766208 \times 10^{-19}$ & $\mathrm{C}$ & \\
Planck's constant & $h$ & $6.62607004 \times 10^{-34}$ & $\mathrm{~J} \cdot \mathrm{s}$ & \\
$\hbar$ & $\hbar$ & $1.0545718 \times 10^{-34}$ & $\mathrm{~J} \cdot \mathrm{s}$ & \\
Boltzmann constant & $k_{B}$ & $8.617333262 \times 10^{-5}$ & $\mathrm{eV} \cdot \mathrm{K}^{-1}$ & \\
$k_{B} T$ & $k_{B} T$ & 25.68 & $\mathrm{meV}$ & $T=298 \mathrm{~K}$ \\
\hline
\end{tabular}

Bahder used modified Luttinger parameters [47, Eq. A3]. In the limit of large $E_{g}$, the modified Luttinger parameters $\gamma_{j m}$ equal the Luttinger parameters $\gamma_{j}: \lim _{E_{g} \rightarrow \infty} \gamma_{j m}=\gamma_{j}, j=1,2,3$.

$$
\begin{aligned}
& \gamma_{1 m}=\gamma_{1}-\frac{E_{p}}{3 E_{g}+\Delta_{s o}} \\
& \gamma_{2 m}=\gamma_{2}-\frac{E_{p}}{2\left(3 E_{g}+\Delta_{s o}\right)} \\
& \gamma_{3 m}=\gamma_{3}-\frac{E_{p}}{2\left(3 E_{g}+\Delta_{s o}\right)}
\end{aligned}
$$


Table 2. Material values [52]

\begin{tabular}{|c|c|c|c|c|c|}
\hline parameter & symbol & GaAs & InP & unit & remarks \\
\hline Luttinger parameter & $\gamma_{1}$ & 6.98 & 5.08 & & \\
\hline Luttinger parameter & $\gamma_{2}$ & 2.06 & 1.60 & & \\
\hline Luttinger parameter & $\gamma_{3}$ & 2.93 & 2.10 & & \\
\hline modified Luttinger parameter & $\gamma_{1 m}$ & 0.73 & 0.11 & & $T=298 \mathrm{~K}$ \\
\hline modified Luttinger parameter & $\gamma_{2 m}$ & -1.06 & -0.88 & & $T=298 \mathrm{~K}$ \\
\hline modifed Luttinger parameter & $\gamma_{3 m}$ & -0.19 & -0.38 & & $T=298 \mathrm{~K}$ \\
\hline band gap & $E_{g}$ & 1.519 & 1.4236 & $\mathrm{eV}$ & $T=0 \mathrm{~K}$ \\
\hline band gap & $E_{g}$ & 1.423 & 1.354 & $\mathrm{eV}$ & $T=298 \mathrm{~K}$ \\
\hline conduction band coupling & $A_{p}$ & -14.7 & - & $\mathrm{eV} \AA^{2}$ & \\
\hline matrix element & $E_{p}$ & 28.8 & 20.7 & $\mathrm{eV}$ & \\
\hline matrix element & $P_{o}^{2}$ & 3.795 & 3.217 & $\mathrm{eV}^{2}$ & $P_{o}^{2}=E_{p} / 2$ \\
\hline spin orbit splitting & $\Delta_{s o}$ & 0.341 & 0.108 & $\mathrm{eV}$ & \\
\hline conduction band & $m_{c}$ & 0.067 & 0.0795 & $m_{o}$ & $T=0 \mathrm{~K}$ \\
\hline conduction band & $m_{c}$ & 0.0635 & $0.075^{\dagger}$ & $m_{o}$ & $T=298 \mathrm{~K}$ \\
\hline heavy hole & $m_{H H}$ & 0.35 & 0.53 & $m_{o}$ & $T=298 \mathrm{~K}$ \\
\hline light hole & $m_{L H}$ & 0.083 & 0.12 & $m_{o}$ & $T=298 \mathrm{~K}$ \\
\hline$E_{g}$ deformation potential & $a$ & -8.33 & -6.6 & $\mathrm{eV}$ & $a=a_{c}+a_{v}$ \\
\hline valence band deformation potential & $a_{v}$ & -1.16 & -0.6 & $\mathrm{eV}$ & \\
\hline deformation potential & $b$ & $-2.0_{-1.9}^{+0.34}$ & $-2.0_{-0.0}^{+1.0}$ & $\mathrm{eV}$ & note wide range \\
\hline shear deformation potential & $d$ & $-4.8_{-1.2}^{+2.1}$ & $-5.0_{-0.0}^{+0.8}$ & $\mathrm{eV}$ & note range \\
\hline stiffness & $c_{11}$ & 118.0 & 102.2 & $\mathrm{GPa}$ & crystal coordinate \\
\hline stiffness & $c_{12}$ & 53.5 & 57.3 & $\mathrm{GPa}$ & crystal coordinate \\
\hline stiffness & $c_{44}$ & 59.0 & 44.2 & $\mathrm{GPa}$ & crystal coordinate \\
\hline stiffness & $c_{11}^{\prime}, c_{22}^{\prime}$ & 144.75 & 123.95 & $\mathrm{GPa}$ & $\{110\}$ facet \\
\hline stiffness & $c_{33}^{\prime}$ & 118.0 & 102.2 & $\mathrm{GPa}$ & $\{110\}$ facet \\
\hline stiffness & $c_{12}^{\prime}$ & 26.750 & 35.55 & $\mathrm{GPa}$ & $\{110\}$ facet \\
\hline stiffness & $c_{13}^{\prime}, c_{23}^{\prime}$ & 53.5 & 57.3 & $\mathrm{GPa}$ & $\{110\}$ facet \\
\hline stiffness & $c_{44}^{\prime}, c_{55}^{\prime}$ & 59.0 & 44.2 & $\mathrm{GPa}$ & $\{110\}$ facet \\
\hline stiffness & $c_{66}^{\prime}$ & 32.250 & 24.450 & $\mathrm{GPa}$ & $\{110\}$ facet \\
\hline
\end{tabular}

$\dagger$ The room temperature value for InP was determined by selecting $A_{p}=-9.98$ to match the recommended $T=0$ value of 0.0795 [52, pg 5829], and using this value for $A_{p}$ in Eq. (6). Using $A_{p}=0$ gives values for $m_{c}$ of 0.088 and 0.083 at $T=0 \mathrm{~K}$ and $T=298 \mathrm{~K}$ for InP. 


\section{B. Bloch Factors and Matrix Elements}

The basis functions (Enders identifies these functions as Bloch factors [48, pg 544]) used by Bahder [45] are

$$
\begin{aligned}
& C B_{1}: \quad|S\rangle|\downarrow\rangle \\
& C B_{2}: \quad|S\rangle|\uparrow\rangle \\
& L H_{1}: \frac{-i}{\sqrt{6}}(|X\rangle+i|Y\rangle)|\downarrow\rangle+i \sqrt{\frac{2}{3}}|Z\rangle|\uparrow\rangle \quad L H_{2}: \frac{i}{\sqrt{6}}(|X\rangle-i|Y\rangle)|\uparrow\rangle+i \sqrt{\frac{2}{3}}|Z\rangle|\downarrow\rangle \\
& H H_{1}: \frac{i}{\sqrt{2}}(|X\rangle+i|Y\rangle)|\uparrow\rangle \quad H H_{2}: \frac{-i}{\sqrt{2}}(|X\rangle-i|Y\rangle)|\downarrow\rangle \\
& S O_{1}: \frac{-i}{\sqrt{3}}(|X\rangle-i|Y\rangle)|\uparrow\rangle+\frac{i}{\sqrt{3}}|Z\rangle|\downarrow\rangle \quad S O_{2}: \frac{-i}{\sqrt{3}}(|X\rangle+i|Y\rangle)|\downarrow\rangle-\frac{i}{\sqrt{3}}|Z\rangle|\uparrow\rangle .
\end{aligned}
$$

These Bloch factors are defined for $\boldsymbol{k}$ along the $z$ direction and the $x, y$, and $z$ directions are aligned along the [100], [010], and [001] directions of the crystal coordinate system.

The rate of transitions between two states $\left|\Psi_{1}\right\rangle$ and $\left|\Psi_{2}\right\rangle$ is proportional to $|M|^{2}$, the magnitude squared of the momentum matrix element $M$, with

$$
M=\left\langle\Psi_{1}|\boldsymbol{\varepsilon} \cdot \boldsymbol{p}| \Psi_{2}\right\rangle,
$$

where $\varepsilon$ is a unit vector that gives the polarization of the electromagnetic wave and $\boldsymbol{p}$ is the momentum operator. See Appendix C. For an electromagnetic wave that is polarized along the $x$ direction, $M=\left\langle\Psi_{1}\left|p_{x}\right| \Psi_{2}\right\rangle$.

The non-zero momentum matrix elements that connect the conduction band and the valence band are $\left\langle S\left|p_{x}\right| X\right\rangle,\left\langle S\left|p_{y}\right| Y\right\rangle$, and $\left\langle S\left|p_{z}\right| Z\right\rangle$. The non-zero spin matrix elements are $\langle\downarrow \mid \downarrow\rangle$ and $\langle\uparrow \mid \uparrow\rangle$. These selection rules plus the Bloch factors for the conduction and valence bands are required to calculate $L_{\hat{\boldsymbol{h}}}$ and $L_{\hat{\boldsymbol{v}}}$ and thus $D O P_{\hat{\boldsymbol{h}} \times \hat{\boldsymbol{v}}}$, Eq. (3).

\section{Transition Rate}

This Appendix follows Chapters 13 and 22 of Gasiorowicz's book, Quantum Physics [56].

The electric $\boldsymbol{E}$ and magnetic $\boldsymbol{B}$ fields are required to describe an electromagnetic field. The coupled $\boldsymbol{E}$ and $\boldsymbol{B}$ components of an electromagnetic field can be combined into a vector potential $\boldsymbol{A}$. Under certain conditions on the charge and current densities, a simple wave equation for the vector potential $\boldsymbol{A}$ can be obtained. The vector potential as a travelling wave is

$$
\boldsymbol{A}(\boldsymbol{r}, t)=\sqrt{\frac{2 \pi c^{2} \mathcal{N} \hbar}{\omega \mathcal{V}}} \boldsymbol{\varepsilon} \exp i\left(\boldsymbol{k}_{\boldsymbol{\varepsilon}} \cdot \boldsymbol{r}-\omega t\right)
$$

where $\varepsilon$ is a unit vector that defines the polarization of the electromagnetic field, $\mathcal{N}$ is the refractive index, and $\mathcal{V}$ is the volume of interest.

The classical force on a electron of reduced mass $\mu$ is given by the Lorentz force equation

$$
\mu \frac{d^{2} \boldsymbol{r}}{d t^{2}}=-e\left(\boldsymbol{E}+\frac{\boldsymbol{v}}{c} \times \boldsymbol{B}\right) .
$$

The Lorentz force equation is derivable if the Hamiltonian is taken as

$$
H=\frac{1}{2 \mu}\left(p+\frac{e}{c} A\right)^{2}
$$


i.e., replace $\boldsymbol{p}$ with $\boldsymbol{p}+\boldsymbol{e} \boldsymbol{A} / c$. This Hamiltonian can be used with time-dependent perturbation theory to calculate the probability of a transition between two optically connected states.

The probability per unit time of a transition is

$$
\Gamma=2 \pi \hbar \frac{2 \pi e^{2} \mathcal{N}}{\mu^{2} \hbar \omega \mathcal{V}}\left|\left\langle\Psi_{1}\left|e^{i \boldsymbol{k}_{\varepsilon} \cdot \boldsymbol{r}} \boldsymbol{\varepsilon} \cdot \boldsymbol{p}\right| \Psi_{2}\right\rangle\right|^{2} .
$$

The total transition rate is determined by summing the probability over all states, modes, and polarizations. The total transition rate $\mathcal{R}$ can be written as

$$
\mathcal{R}=\frac{2 \pi}{\hbar}|M|^{2} \rho(E)
$$

where $\rho(E)$ is the density of final states and the equation is called Fermi's Golden Rule. The matrix element $M$ describes the optical connection between the states and is given by

$$
M=\left\langle\Psi_{1}\left|e^{i \boldsymbol{k}_{\varepsilon} \cdot \boldsymbol{r}} \boldsymbol{\varepsilon} \cdot \boldsymbol{p}\right| \Psi_{2}\right\rangle .
$$

For transitions in a crystal, the wave vector of the radiation $\boldsymbol{k}_{\boldsymbol{\varepsilon}}$ is significantly smaller than the crystal momenta $\boldsymbol{k}$ of the carriers in the crystal that are involved in the transition, and can be ignored. Both $\Psi_{1}$ and $\Psi_{2}$, which are Bloch functions, contain multiplicative factors of $\exp \left(i \boldsymbol{k}_{\mathbf{1}} \cdot \boldsymbol{r}\right)$ and $\exp \left(i \boldsymbol{k}_{\mathbf{2}} \cdot \boldsymbol{r}\right)$, which leads to a Dirac delta function of $\delta\left(-\boldsymbol{k}_{\mathbf{1}}+\boldsymbol{k}_{\mathbf{2}}+\boldsymbol{k}_{\boldsymbol{\varepsilon}}\right) \approx \delta\left(-\boldsymbol{k}_{\mathbf{1}}+\boldsymbol{k}_{\mathbf{2}}\right)$ in the matrix element $M$ to express conservation of $\boldsymbol{k}$ [57]. The result is that

$$
M=\left\langle\Psi_{1}|\boldsymbol{\varepsilon} \cdot \boldsymbol{p}| \Psi_{2}\right\rangle,
$$

a result that is needed [57, Eq. 4]. The relative rates of emission for two orthogonal polarizations can be determined from the preceding expressions.

For parabolic bands, $\boldsymbol{k}$ and $\boldsymbol{p}$ are parallel. The dot product with the polarization vector ensures that the light is a transverse wave. This means that to calculate the DOP, only $\boldsymbol{k}$ that are in the plane of the measurement need to be considered (in the approximate analysis presented in this manuscript where the luminescences along principle directions are used as proxies for the integrated luminescence).

Baym [58, pg 282] shows for an isolated atom with Hamiltonian $H_{o}$ that the matrix element can be written in terms of the dipole moment operator $\left\langle\Psi_{1}|\boldsymbol{\varepsilon} \cdot \boldsymbol{r}| \Psi_{2}\right\rangle$. By using $i \hbar \boldsymbol{p}=\left[\boldsymbol{r}, H_{o}\right]$, $M$ can be expressed as:

$$
\begin{aligned}
M & =\frac{1}{i \hbar} \boldsymbol{\varepsilon} \cdot\left\langle\Psi_{1}\left|\boldsymbol{r} H_{o}-H_{o} \boldsymbol{r}\right| \Psi_{2}\right\rangle \\
& =\frac{\left(E_{2}-E_{1}\right)}{i \hbar}\left\langle\Psi_{1}|\boldsymbol{\varepsilon} \cdot \boldsymbol{r}| \Psi_{2}\right\rangle,
\end{aligned}
$$

which gives the matrix element in terms of the dipole moment. Only states with a non-zero dipole moment, such as $\langle S|x| X\rangle$, are considered optically coupled. The dipole moment and momentum matrix element are perhaps easier to visualize and understand than a density matrix approach [59]. Regardless, one must exercise caution to ensure that selection rules are followed.

\section{Rotation Matrix}

The defining features of tensors, vectors, and scalars are how they change under transformation of the coordinate system [53].

If scalars, vectors, and tensors represent physical objects, then magnitudes and directions should be independent of the choice of a coordinate system. Thus the components of a vector or a tensor must follow rules for transformations between coordinate systems. 
Consider two Cartesian coordinate systems $(\boldsymbol{i}, \boldsymbol{j}, \boldsymbol{k})$ and $\left(\boldsymbol{i}^{\prime}, \boldsymbol{j}^{\prime}, \boldsymbol{k}^{\prime}\right)$ with the same origin, but with the axes not necessarily aligned. The components of a vector $\boldsymbol{A}$ in the primed coordinate system are related to the components in the unprimed coordinate system by $\left(A_{x^{\prime}}, A_{y^{\prime}}, A_{z^{\prime}}\right)^{T}=$ $[R] \times\left(A_{x}, A_{y}, A_{z}\right)^{T}$ where $[R]$ is the rotation matrix and is a $3 \times 3$ matrix. The rotation matrix $[R]$ is $[60, \mathrm{pg} 6]$

$$
[R]=\left[\begin{array}{lll}
\boldsymbol{i}^{\prime} \cdot \boldsymbol{i} & \boldsymbol{i}^{\prime} \cdot \boldsymbol{j} & \boldsymbol{i}^{\prime} \cdot \boldsymbol{k} \\
\boldsymbol{j}^{\prime} \cdot \boldsymbol{i} & \boldsymbol{j}^{\prime} \cdot \boldsymbol{j} & \boldsymbol{j}^{\prime} \cdot \boldsymbol{k} \\
\boldsymbol{k}^{\prime} \cdot \boldsymbol{i} & \boldsymbol{k}^{\prime} \cdot \boldsymbol{j} & \boldsymbol{k}^{\prime} \cdot \boldsymbol{k}
\end{array}\right]=\left[\begin{array}{lll}
a_{11} & a_{12} & a_{13} \\
a_{21} & a_{22} & a_{23} \\
a_{31} & a_{32} & a_{33}
\end{array}\right] .
$$

\section{E. Euler's Angles}

A rigid body in a $3 \mathrm{D}$ space requires three angles to specify its orientation relative to a frame of reference $(\boldsymbol{i}, \boldsymbol{j}, \boldsymbol{k})$. Euler's angles are one method to specify the orientation of a body with respect to a frame of reference. There are several conventions for specifying Euler's angles. The convention employed here is: Start with the $\left(\boldsymbol{i}^{\prime}, \boldsymbol{j}^{\prime}, \boldsymbol{k}^{\prime}\right)$ and the $(\boldsymbol{i}, \boldsymbol{j}, \boldsymbol{k})$ coordinate systems aligned. Rotate counter-clockwise by an angle $\alpha$ about the $\boldsymbol{k}$ axis to obtain the $\left(\boldsymbol{i}^{\prime}, \boldsymbol{j}^{\prime}, \boldsymbol{k}^{\prime}\right)$ basis. Rotate clockwise by an angle $\beta$ about the $\boldsymbol{j}^{\prime}$ axis to obtain the $\left(\boldsymbol{i}^{\prime \prime}, \boldsymbol{j}^{\prime \prime}, \boldsymbol{k}^{\prime \prime}\right)$ basis. Finally, rotate counter-clockwise by an angle $\gamma$ about the $\boldsymbol{k}^{\prime \prime}$ axis. These three rotations will align the $\left(\boldsymbol{i}^{\prime \prime \prime}, \boldsymbol{j}^{\prime \prime \prime}, \boldsymbol{k}^{\prime \prime \prime}\right)$ triad in an arbitrary direction in space. If $\left[R_{z}(\theta)\right]$ is a rotation matrix for rotation of $\theta$ about the $\boldsymbol{k}$ axis, then the rotation matrix for the three rotations is $\left[R_{z^{\prime \prime}}(\gamma)\right] \times\left[R_{y^{\prime}}(\beta)\right] \times\left[R_{z}(\alpha)\right]$. The clockwise and counter-clockwise directions are defined from the perspective of an observer positioned on the positive side of the axis of rotation and facing the origin [60, pp 7 and 10-11].

$$
\left[R_{z}(\alpha)\right]=\left[\begin{array}{ccc}
\cos (\alpha) & \sin (\alpha) & 0 \\
-\sin (\alpha) & \cos (\alpha) & 0 \\
0 & 0 & 1
\end{array}\right] \quad\left[R_{y^{\prime}}(\beta)\right]=\left[\begin{array}{ccc}
\cos (\beta) & 0 & -\sin (\beta) \\
0 & 1 & 0 \\
\sin (\beta) & 0 & \cos (\beta)
\end{array}\right]
$$

The intermediate steps in the rotations need not be written down. Given that the vector $\boldsymbol{A}$ has components $\left(A_{x}, A_{y}, A_{z}\right)$ in the $(\boldsymbol{i}, \boldsymbol{j}, \boldsymbol{k})$ coordinate system, then the components of the vector $\boldsymbol{A}$ in the $\left(\boldsymbol{i}^{\prime \prime \prime}, \boldsymbol{j}^{\prime \prime \prime}, \boldsymbol{k}^{\prime \prime \prime}\right)$ coordinate system are

$$
\left[\begin{array}{c}
A_{x^{\prime \prime \prime}} \\
A_{y^{\prime \prime \prime}} \\
A_{z^{\prime \prime \prime}}
\end{array}\right]=\left[R_{z^{\prime \prime}}(\gamma)\right] \times\left[R_{y^{\prime}}(\beta)\right] \times\left[R_{z}(\alpha)\right]\left[\begin{array}{c}
A_{x} \\
A_{y} \\
A_{z}
\end{array}\right] .
$$

The inverse transformation is needed. Given some vector $\boldsymbol{A}$ with components $\left(A_{x^{\prime \prime \prime}}, A_{y^{\prime \prime \prime}}, A_{z^{\prime \prime \prime}}\right)$ in the $\left(\boldsymbol{i}^{\prime \prime \prime}, \boldsymbol{j}^{\prime \prime \prime}, \boldsymbol{k}^{\prime \prime \prime}\right)$ coordinate system, the components of vector $\boldsymbol{A}$ in the $(\boldsymbol{i}, \boldsymbol{j}, \boldsymbol{k})$ coordinate system are

$$
\left[\begin{array}{c}
A_{x} \\
A_{y} \\
A_{z}
\end{array}\right]=\left[R_{z}(-\alpha)\right] \times\left[R_{y^{\prime}}(-\beta)\right] \times\left[R_{z^{\prime \prime}}(-\gamma)\right] \times\left[\begin{array}{c}
A_{x^{\prime \prime \prime}} \\
A_{y^{\prime \prime \prime}} \\
A_{z^{\prime \prime \prime}}
\end{array}\right]
$$

Since $\left[R_{z}(-\alpha)\right]=\left[R_{z}(\alpha)\right]^{T}$ and $[R]^{T}[R]=[R][R]^{T}=[\boldsymbol{I}]$ where $[\boldsymbol{I}]$ is the identity matrix (i.e., rotation is a unitary transformation), negative rotations in the inverse order undo the transformation. 


\section{F. Representation Quadric [53]}

The three components of a vector $\left(A_{1}, A_{2}, A_{3}\right)$ transform under rotation of the basis (i.e, under rotation of the coordinate system) as

$$
A_{m}^{\prime}=a_{m n} A_{n}=\sum_{n=1}^{3} a_{m n} A_{n}
$$

where $a_{m n}=\hat{\boldsymbol{x}}_{\boldsymbol{m}}^{\prime} \cdot \hat{\boldsymbol{x}}_{\boldsymbol{n}}$ [53, pg 10]. $\left(A_{1}^{\prime}, A_{2}^{\prime}, A_{3}^{\prime}\right)$ are the components of $\boldsymbol{A}$ in the rotated basis $\left(\hat{x}_{1}^{\prime}, \hat{x}_{2}^{\prime}, \hat{x}_{3}^{\prime}\right)$ and $\left(A_{1}, A_{2}, A_{3}\right)$ are the components of $\boldsymbol{A}$ in the original basis $\left(\hat{x}_{1}, \hat{x}_{2}, \hat{x}_{3}\right)$. Note use of the Einstein summation convention. Note also that Eq. (F.1) can be written in matrix form as $\left(A_{1}^{\prime}, A_{2}^{\prime}, A_{3}^{\prime}\right)^{T}=[R] \times\left(A_{1}, A_{2}, A_{3}\right)^{T}$, as presented in Appendices D and $\mathrm{E}$.

The nine components $T_{i j}$ of a second rank tensor $[T]$ transform under rotation of the basis as $T_{i j}^{\prime}=a_{i k} a_{j l} T_{k l}$ where $T_{i j}^{\prime}$ are the nine components in the rotated basis [53,pg 11]. The $a_{n m}$ are as defined for rotation of a vector.

Consider the equation for a second degree surface (i.e., a quadric, [53, pg 16]) where the coefficients are 'symmetric' (i.e., $S_{m n}=S_{n m}$ ):

$$
S_{m n} x_{m} x_{n}=1 .
$$

Rotate the basis and invert the rotation using the orthogonal property of rotation (i.e., $\hat{\boldsymbol{x}}_{\boldsymbol{i}}^{\prime} \cdot \hat{\boldsymbol{x}}_{j}^{\prime}=\delta_{i j}=a_{i k} a_{j k}$, and $a_{k i} a_{k j}=\delta_{i j}$ since rotation is an unitary transformation) to obtain $\hat{\boldsymbol{x}}_{\boldsymbol{m}}=a_{p m} \hat{\boldsymbol{x}}_{\boldsymbol{p}}^{\prime}$ and $\hat{\boldsymbol{x}}_{\boldsymbol{n}}=a_{p n} \hat{\boldsymbol{x}}_{\boldsymbol{p}}^{\prime}$. Substitute the expressions for the components of $\hat{\boldsymbol{x}}$ into the equation for a second degree surface (i.e., into the equation for the quadric):

$$
S_{m n} x_{m} x_{n}=S_{m n} a_{p m} x_{p}^{\prime} a_{q n} x_{q}^{\prime}=a_{p m} a_{q n} S_{m n} x_{p}^{\prime} x_{q}^{\prime}=S_{p q}^{\prime} x_{p}^{\prime} x_{q}^{\prime} .
$$

The coefficients of the quadric transform the same as a second rank tensor [53, pg 16]. Since the coefficients of the quadric are symmetric, $S_{p q}=S_{q p}$, the components $T_{m n}$ of a symmetric, second rank tensor $[T]$ transform the same as the coefficients of a quadric. A quadric thus provides a means to visualize a symmetric, second rank tensor and the surface $T_{m n} x_{m} x_{n}=1$ is called the representation quadric of the tensor [T] [53, pg 17].

The equation for the quadric surface, Eq. (F.2), can be written as a matrix equation

$$
[x][S][x]^{T}=\left[\begin{array}{lll}
x_{1} & x_{2} & x_{3}
\end{array}\right]\left[\begin{array}{lll}
S_{1} & S_{6} & S_{5} \\
S_{6} & S_{2} & S_{4} \\
S_{5} & S_{4} & S_{3}
\end{array}\right]\left[\begin{array}{c}
x_{1} \\
x_{2} \\
x_{3}
\end{array}\right]=1,
$$

with the matrix $[S]$ written in a Voigt notation to reduce the number of subscripts.

Thus by analogy, a symmetric, second rank tensor can be written in matrix form. The properties (e.g., principal axes, eigenvalues, eigenvectors, transformation, and principal invariants, which are the trace, the determinate, and $\left.S_{11} S_{22}+S_{22} S_{33}+S_{11} S_{33}-S_{12}^{2}-S_{23}^{2}-S_{13}^{2}\right)$ ) of a quadric are known. Thus, by analogy, the properties of a symmetric, second rank tensor are also known.

The transformation for a symmetric, second rank tensor, Eq. (F.3), can be calculated as $[S]=[R]^{T}\left[S^{\prime}\right][R]$, where $[R]=[R(\alpha, \beta, \gamma)]=\left[R_{z^{\prime \prime}}(\gamma)\right] \times\left[R_{y^{\prime}}(\beta)\right] \times\left[R_{z}(\alpha)\right]$ is the full rotation matrix. Since rotation is a unitary transformation (i.e., $[R][R]^{T}=[R]^{T}[R]=[\boldsymbol{I}]$ ), the inverse transformation is $\left[S^{\prime}\right]=[R][S][R]^{T}[53$, pg 158]. 